\title{
GENERAL BOUNDARY CONDITIONS FOR AN ORDINARY LINEAR DIFFERENTIAL SYSTEM
}

BY

RANDAL H. COLE

1. Introduction. Various types of boundary conditions have been associated with the matrix equation

$$
\mathfrak{Y}^{\prime}=\mathfrak{A}(x, \lambda) \mathfrak{Y}+\mathfrak{B}(x),
$$

where $\mathfrak{A}(x, \lambda)$ is a square matrix of order $n$. Most of these are special cases of the general condition

$$
\sum_{h} \mathfrak{W}^{(h)}(\lambda) \mathfrak{Y}\left(a_{h}, \lambda\right)+\int_{a}^{b} \mathfrak{W}(x, \lambda) \mathfrak{Y}(x, \lambda) d x=\mathfrak{C}(\lambda),
$$

where the matrix $\mathfrak{W}^{(h)}(\lambda)$ is free from $x$ and $\left\{a_{h}\right\}$ is a finite or infinite set of points on a fundamental interval $[a, b]$. Tamarkin [5] has considered the general case, but many of his results were obtained by limiting the discussion to the situation where $\mathfrak{W}(x, \lambda)=\mathfrak{D}$ or where no boundary points exist in the interior of the fundamental interval. In particular, he did not define the adjoint system for the general case. Wilder [9] and Cole [1] have treated the case of a finite set of points and no integral term. Langer [2] has developed the theory associated with a finite set of boundary points in a complex domain. Whyburn has made substantial contributions to the problem, including a summary $[6 ; 8]$ of known results. He has also shown [7] that the condition (1.2) is, in a certain sense, equivalent to

$$
\mathfrak{A}(\lambda) \mathfrak{Y}(a, \lambda)+\mathfrak{B}(\lambda) \mathfrak{Y}(b, \lambda)+\int_{a}^{b} \mathfrak{F}(x, \lambda) \mathfrak{Y}(x, \lambda) d x=\mathfrak{C}(\lambda)
$$

It will be seen, however, that the condition (1.2) is distinct from(1.3) in the sense that the two conditions lead to distinct adjoint boundary relations.

Interface conditions of the type

$$
\mathfrak{Y}\left(a_{h}^{+}, \lambda\right)-B^{(h)}(\lambda) \mathfrak{Y}\left(a_{h}^{-}, \lambda\right)=\mathfrak{D}
$$

have been considered by Stallard [4]. Such conditions are not included in (1.2), but similar conditions do appear in the adjoint system. In an earlier paper [1], it is shown that interface conditions are the adjoint counterparts of that portion

Received by the editors March 4, 1963. 
of (1.2) which applies to the discrete points $\left\{a_{h}\right\}$. When discrete and continuous relations are applied together, as in (1.2), it is of particular interest to observe the distinct way in which each is reflected in the adjoint system.

The definition of the adjoint system, given in $\$ 4$, is a significant feature of the present development. It is different from the adjoint system defined by Whyburn [7], although the Green's matrix defined here (when $m=2$ ) is identical with that defined by Whyburn. It is significant that Green's matrix provides a solution in integral form for the nonhomogeneous adjoint system as well as for the original system. Green's matrix is itself a formal solution of both the given system and the adjoint system. These two properties of a Green's function are familiar from classical developments.

A second significant feature of this discussion is the determination of the structure of Green's matrix. It is patterned after the corresponding development in the $m$-point case [1] and, as in that case, the analysis is vital to the development of the expansion theory associated with the system.

2. The differential system. The system to be considered is

$$
\begin{gathered}
\mathfrak{Y}^{\prime}=\mathfrak{H}(x, \lambda) \mathfrak{Y}, \\
\sum_{h=1}^{m} \mathfrak{W}^{(h)}(\lambda) \mathfrak{Y}\left(a_{h}, \lambda\right)+\int_{a}^{b} \mathfrak{W}(x, \lambda) \mathfrak{Y}(x, \lambda) d x=\mathfrak{D},
\end{gathered}
$$

where $\mathfrak{A}(x, \lambda)$ is continuous in both variables, and $\mathfrak{B}^{(h)}(\lambda), h=1,2, \cdots, m$, and $\mathfrak{W}(x, \lambda)$ are matrices whose components are polynomials in the parameter $\lambda$. In the case of $\mathfrak{B}(x, \lambda)$ the coefficients of the polynomials are indefinitely differentiable functions of $x$. The set of points $a_{h}, h=1,2, \cdots, m$, is such that $a_{h}<a_{h+1}$, with $a_{1}=a$ and $a_{m}=b$. The two terms on the left of (2.1b) can be conveniently combined into one by using a Stieltjes integral. Thus, let $\mathfrak{F}_{1}(x, \lambda)$ be defined as constant on $[a, b]$ except at the points $a_{1}, a_{2}, \cdots, a_{m}$. At these points it is such that

with

$$
\mathfrak{F}_{1}\left(a_{h}^{+}, \lambda\right)-\mathfrak{F}_{1}\left(a_{h}^{-}, \lambda\right)=\mathfrak{W}^{(h)}(\lambda),
$$

$$
\mathfrak{F}_{1}\left(a_{1}, \lambda\right)=\mathfrak{F}_{1}\left(a_{1}^{-}, \lambda\right)=\mathfrak{D} \text { and } \mathfrak{F}_{1}\left(a_{m}, \lambda\right)=\mathfrak{F}_{1}\left(a_{m}^{+}, \lambda\right) .
$$

Let $\mathfrak{F}_{2}(x, \lambda)$ be defined by

$$
\mathfrak{F}_{2}(x, \lambda)=\int_{a}^{x} \mathfrak{W}(t, \lambda) d t, \quad a \leqq x \leqq b .
$$

Then, if

$$
\mathfrak{F}(x, \lambda)=\mathfrak{F}_{1}(x, \lambda)+\mathfrak{F}_{2}(x, \lambda),
$$

the condition (2.1b) may be written as 


$$
\int_{a}^{b} d \mathfrak{F}(x, \lambda) \mathfrak{Y}(x, \lambda)=\mathfrak{D}
$$

The symbol on the left of (2.3) represents a matrix whose component in the $i$ th row and $j$ th column is

$$
\sum_{k=1}^{n} \int_{a}^{b} y_{k j}(x, \lambda) d f_{i k}(x, \lambda)
$$

The general vector solution of (2.1a) is given by

$$
\mathfrak{y}=\mathfrak{Y}(x, \lambda) \mathfrak{c}(\lambda),
$$

where $\mathfrak{Y}(x, \lambda)$ is a specific nonsingular matrix solution of $(2.1 \mathrm{a})$, and $\mathfrak{c}(\lambda)$ is an arbitrary vector independent of $x$. This satisfies the boundary condition $(2.1 \mathrm{~b})$ if and only if

$$
\mathfrak{D}(\lambda) \mathfrak{c}(\lambda)=\mathfrak{v},
$$

where $\mathfrak{D}(\lambda)$, the characteristic matrix, is given by

$$
\mathfrak{D}(\lambda)=\int_{a}^{b} d \mathfrak{F}(x, \lambda) \mathfrak{Y}(x, \lambda) .
$$

Solutions of (2.5) exist if and only if

$$
D(\lambda)=0,
$$

where $D(\lambda)=|\mathfrak{D}(\lambda)|$. Values of $\lambda$ satisfying (2.7) are characteristic values, and corresponding solutions of the system are characteristic solutions. If, for a specific characteristic value, $\mathfrak{D}(\lambda)$ is of rank $n-r$, the system is compatible to the order $r$, in the sense that there exist $r$ linearly independent characteristic solutions.

3. Green's matrix. Whyburn [7] has obtained the Green's matrix for system (2.1) when $m=2$. The derivation given here will therefore be brief, since the case for a general integer $m$ is not essentially different from the special case. If $\mathfrak{Y}(x, \lambda)$ represents a specific nonsingular matrix solution of $(2.1 \mathrm{a})$, it is found by the method of variation of parameters that

$$
\mathfrak{B}(x, \lambda)=\mathfrak{Y}(x, \lambda) \mathfrak{C}+\int_{a}^{x} \mathfrak{Y}(x, \lambda) \mathfrak{Y}^{-1}(s, \lambda) \mathfrak{B}(s) d s
$$

is the general solution of the nonhomogeneous equation (1.1). If this is substituted in (2.1b) and if $\mathfrak{D}(\lambda)$, defined by (2.6) is nonsingular, it is readily inferred that

$$
\mathfrak{C}=-\mathfrak{D}^{-1}(\lambda) \int_{a}^{b} d \mathfrak{F}(t, \lambda) \int_{a}^{t} \mathfrak{Y}(t, \lambda) \mathfrak{Y}^{-1}(s, \lambda) \mathfrak{B}(s) d s .
$$

Hence, the solution of the nonhomogeneous boundary system is given by 


$$
\begin{aligned}
\mathfrak{B}(x, \lambda)= & \left.-\int_{t=a}^{b} \int_{s=a}^{t} \mathfrak{Y}(x, \lambda) \mathfrak{D}^{-1}(\lambda) d \mathfrak{F}(t, \lambda) \mathfrak{Y}(t, \lambda) \mathfrak{Y}\right)^{-1}(s, \lambda) \mathfrak{B}(s) d s \\
& \left.+\int_{a}^{x} \mathfrak{Y}(x, \lambda) \mathfrak{Y}\right)^{-1}(s, \lambda) \mathfrak{B}(s) d s .
\end{aligned}
$$

If the identity matrix, in the form

$$
\int_{a}^{b} \mathfrak{D}^{-1}(\lambda) d \mathfrak{F}(t, \lambda) \mathfrak{Y}(t, \lambda),
$$

is inserted between $\mathfrak{Y}(x, \lambda)$ and $\mathfrak{Y}^{-1}(s, \lambda)$ in the second integral and if the order of integration is reversed in the first integral, we get

$$
\begin{aligned}
\mathfrak{B}(x, \lambda)= & -\int_{s=a}^{b} \int_{t=s}^{b} \mathfrak{Y}(x, \lambda) \mathfrak{D}^{-1}(\lambda) d \mathfrak{F}(t, \lambda) \mathfrak{Y}(t, \lambda) \mathfrak{Y}^{-1}(s, \lambda) \mathfrak{B}(s) d s \\
& +\int_{s=a}^{x} \int_{t=a}^{b} \mathfrak{V}(x, \lambda) \mathfrak{D}^{-1}(\lambda) d \mathfrak{F}(t, \lambda) \mathfrak{Y}(t, \lambda) \mathfrak{Y}^{-1}(s, \lambda) \mathfrak{B}(s) d s .
\end{aligned}
$$

The values of these two integrals over their common domain cancel, so that

$$
\mathfrak{B}(x, \lambda)=\int_{a}^{b} \mathfrak{G}(x, s, \lambda) \mathfrak{B}(s) d s,
$$

where $\mathfrak{G}(x, s, \lambda)$, the Green's matrix, is given by

(3.1) $\mathfrak{G}(x, s, \lambda)=\left\{\begin{aligned} \int_{a}^{s} \mathfrak{Y}(x, \lambda) \mathfrak{D}^{-1}(\lambda) d \mathfrak{F}(t, \lambda) \mathfrak{Y}(t, \lambda) \mathfrak{Y}^{-1}(s, \lambda), & s<x, \\ -\int_{s}^{b} \mathfrak{Y}(x, \lambda) \mathfrak{D}^{-1}(\lambda) d \mathfrak{F}(t, \lambda) \mathfrak{Y}(t, \lambda) \mathfrak{Y}^{-1}(s, \lambda), & s>x .\end{aligned}\right.$

The properties of Green's matrix will be listed in the form of a theorem. When $m=2$, they are consistent with those given by Whyburn [7].

THEOREM 1. Green's matrix has the following properties:

(1) It is continuous in $x$ and $s$ except when $x=s$ and

$$
s=a_{1}, a_{2}, \cdots, a_{m} .
$$

(2) It has a unit discontinuity at $x=s$, that is,

$$
\mathfrak{G}\left(s^{+}, s, \lambda\right)-\mathfrak{G}\left(s^{-}, s, \lambda\right)=\mathfrak{J} .
$$

(3) At the boundary points, the discontinuities are given by

$$
\mathfrak{G}\left(x, a_{h}^{+}, \lambda\right)-\mathfrak{G}\left(x, a_{h}^{-}, \lambda\right)=\mathfrak{Y}(x, \lambda) \mathfrak{D}^{-1}(\lambda) \mathfrak{W}^{(h)}(\lambda), h=1,2, \cdots, m,
$$

with the definition, 


$$
\mathfrak{G}\left(x, a_{1}^{-}, \lambda\right)=\mathfrak{b}\left(x, a_{m}^{+}, \lambda\right)=\mathfrak{D} \text {. }
$$

(4) For each fixed $s, \mathfrak{G}(x, s, \lambda)$ is a formal solution of system (2.1).

Proof. The first three properties follow directly from the definition of $\mathfrak{G}(x, s, \lambda)$. The definition, given under property (3), of left and right hand limits for $\mathfrak{G}(x, s, \lambda)$ at $a_{1}$ and $a_{m}$, respectively, is merely a notational convenience.

To establish property (4), we note first that $\mathfrak{G}(x, s, \lambda)$ is a formal solution of (2.1a) by virtue of having $\mathfrak{Y}(x, \lambda)$ as its left hand factor. It fails to be a true solution because of the discontinuity at $x=s$. If $\mathfrak{F}(x, s, \lambda)$ is substituted in the left side of (2.1b) or (2.3), we have

$$
\begin{aligned}
\int_{a}^{b} d \mathfrak{F}(x, \lambda) \mathfrak{F}(x, s, \lambda)= & \int_{a}^{s} d \mathfrak{F}(x, \lambda)\left\{-\int_{s}^{b} \mathfrak{Y}(x, \lambda) \mathfrak{D}^{-1}(\lambda) d \mathfrak{F}(t, \lambda) \mathfrak{Y}(t, \lambda) \mathfrak{Y}^{-1}(s, \lambda)\right\} \\
& \left.+\int_{s}^{b} d \mathfrak{F}(x, \lambda)\left\{\int_{a}^{s} \mathfrak{Y}(x, \lambda) \mathfrak{D}^{-1}(\lambda) d \mathfrak{F}(t, \lambda) \mathfrak{Y}(t, \lambda) \mathfrak{Y}\right)^{-1}(s, \lambda)\right\} .
\end{aligned}
$$

If the expression

$$
\int_{s}^{b} d \mathfrak{F}(x, \lambda)\left\{\int_{s}^{b} \mathfrak{Y}(x, \lambda) \mathfrak{D}^{-1}(\lambda) d \mathfrak{F}(t, \lambda) \mathfrak{Y}(t, \lambda) \mathfrak{Y}^{-1}(s, \lambda)\right\},
$$

is added to and subtracted from the right side of (3.3), we get

$$
\begin{aligned}
\int_{a}^{b} d \mathfrak{F}(x, \lambda) \mathfrak{L}(x, s, \lambda)= & -\mathfrak{D}(\lambda) \mathfrak{D}^{-1}(\lambda) \int_{s}^{b} d \mathfrak{F}(t, \lambda) \mathfrak{Y}(t, \lambda) \mathfrak{Y}^{-1}(s, \lambda) \\
& +\int_{s}^{b} d \mathfrak{F}(x, \lambda) \mathfrak{Y}(x, \lambda) \mathfrak{D}^{-1}(\lambda) \mathfrak{D}(\lambda) \mathfrak{Y}^{-1}(s, \lambda) .
\end{aligned}
$$

Since the right side of this relation is obviously zero, property (4) is established, and the theorem is proved.

4. The adjoint system. The boundary system adjoint to (2.1) is defined to be

$$
\begin{aligned}
& \mathfrak{Z}^{\prime}=-\mathfrak{Z} \mathfrak{Y}(x, \lambda)+\mathfrak{K}(\lambda) \mathfrak{W}(x, \lambda), \\
& \mathfrak{Z}\left(a_{h}^{+}, \lambda\right)-\mathfrak{Z}\left(a_{h}^{-}, \lambda\right)=\mathfrak{H}(\lambda) \mathfrak{W}^{(h)}(\lambda), \quad h=1,2, \cdots, m,
\end{aligned}
$$

where $3\left(a_{1}^{-}, \lambda\right)$ and $\mathcal{Z}\left(a_{m}^{+}, \lambda\right)$ are defined as symbols representing the zero matrix. A solution of this system exists in conjunction with a parametric matrix $\Re(\lambda)$. Because of the conditions (4.1b), any solution is of necessity a solution in the extended sense defined by Stallard [4]. The question of the existence of a vector solution $z(x, \lambda)$ of $(4.1)$ is clearly pertinent. Such a solution will be associated with a parametric vector $\mathfrak{f}(\lambda)$. The general vector solution of the reduced adjoint equation, 


$$
3^{\prime}=-3 \mathfrak{U}(x, \lambda)
$$

is given by

$$
\mathfrak{z}=\mathfrak{c}(\lambda) \mathfrak{Y}^{-1}(x, \lambda),
$$

where $\mathfrak{Y}^{-1}(x, \lambda)$ is the inverse of the matrix $\mathfrak{Y}(x, \lambda)$ in relation $(2.4)$, and $\mathfrak{c}(\lambda)$ is an arbitrary vector. The method of variation of parameters, previously used in developing Green's matrix, yields a particular solution,

$$
\mathfrak{f}(\lambda) \int_{a}^{x} \mathfrak{W}(t, \lambda) \mathfrak{Y}(t, \lambda) d t \mathfrak{Y}^{-1}(x, \lambda),
$$

of equation (4.1a). Thus, the general solution of this equation is

$$
\left.\mathfrak{z}=\mathfrak{c}(\lambda) Y^{-1}(x, \lambda)+\mathfrak{F}(\lambda) \int_{a}^{x} \mathfrak{W}(t, \lambda) \mathfrak{Y}(t, \lambda) d t \mathfrak{Y}\right)^{-1}(x, \lambda) .
$$

The conditions (4.1b) require that $c(\lambda)$ be independently defined on each subinterval $\left(a_{q}, a_{q+1}\right), q=1,2, \cdots, m-1$. Hence, a solution of the adjoint system has the form

$$
\begin{aligned}
& 3(x, \lambda)=\mathfrak{c}_{q}(\lambda) \mathfrak{Y}^{-1}(x, \lambda)+\mathfrak{f}(\lambda) \int_{a}^{x} \mathfrak{W}(t, \lambda) \mathfrak{Y}(t, \lambda) d t \mathfrak{Y}^{-1}(x, \lambda), \\
& a_{q}<x<a_{q+1}, \quad q=1,2, \cdots, m-1 .
\end{aligned}
$$

Substituting in (4.1b) yields the relations

$$
\begin{aligned}
& \mathfrak{c}_{1}(\lambda) \mathfrak{Y}^{-1}\left(a_{1}, \lambda\right)=\mathfrak{f}(\lambda) \mathfrak{M}^{(1)}(\lambda), \\
& {\left[\mathfrak{c}_{2}(\lambda)-\mathfrak{c}_{1}(\lambda)\right] \mathfrak{Y}^{-1}\left(a_{2}, \lambda\right)=\mathfrak{f}(\lambda) \mathfrak{M}^{(2)}(\lambda),} \\
& {\left[\mathfrak{c}_{m-1}(\lambda)-\mathfrak{c}_{m-2}(\lambda)\right] \mathfrak{Y}^{-1}\left(a_{m-1}, \lambda\right)=\mathfrak{f}(\lambda) \mathfrak{W}^{(m-1)}(\lambda),} \\
& -c_{m-1}(\lambda) \mathfrak{Y}^{-1}\left(a_{m}, \lambda\right)-\mathfrak{F}(\lambda) \int_{a}^{b} \mathfrak{W}(t, \lambda) \mathfrak{Y}(t, \lambda) d t \mathfrak{Y}^{-1}\left(a_{m}, \lambda\right)=\mathfrak{F}(\lambda) \mathfrak{P}^{(m)}(\lambda) .
\end{aligned}
$$

Multiplying the first of these relations on the right by $\mathfrak{Y}\left(a_{1}, \lambda\right)$, the second by $\mathfrak{Y}\left(a_{2}, \lambda\right), \cdots$, and the last by $\mathfrak{Y}\left(a_{m}, \lambda\right)$, and adding, we get

$$
\mathfrak{D}=\mathfrak{F}(\lambda)\left\{\sum_{h=1}^{m} \mathfrak{B}^{(h)}(\lambda) \mathfrak{Y}\left(a_{h}, \lambda\right)+\int_{a}^{b} \mathfrak{B}(t, \lambda) \mathfrak{Y}(t, \lambda) d t\right\} .
$$

Hence, a necessary condition for a solution is that

$$
\mathfrak{f}(\lambda) \mathfrak{D}(\lambda)=\mathfrak{D} .
$$

Conversely, if $\mathfrak{D}(\lambda)$ is singular, a nontrivial vector $\mathfrak{f}(\lambda)$ will exist. The use of this vector in (4.3) provides evaluations of $c_{1}(\lambda), \cdots, c_{m-1}(\lambda)$. These are given by 


$$
\mathfrak{c}_{q}(\lambda)=\mathfrak{f}(\lambda) \sum_{h=1}^{q} \mathfrak{W}^{(h)}(\lambda) \mathfrak{Y}\left(a_{h}, \lambda\right), \quad q=1,2, \cdots, m-1 .
$$

Substituting in (4.2), we get, for $x$ on $\left(a_{q}, a_{q+1}\right)$,

$$
\mathfrak{3}(x, \lambda)=\mathfrak{F}(\lambda)\left\{\sum_{h=1}^{q} \mathfrak{B}^{(h)}(\lambda) \mathfrak{Y}\left(a_{h}, \lambda\right)+\int_{a}^{x} \mathfrak{W}(t, \lambda) \mathfrak{Y}(t, \lambda) d t\right\} \mathfrak{Y}^{-1}(x, \lambda) .
$$

This may be written as

$$
\left.\mathfrak{3}(x, \lambda)=\mathfrak{F}(\lambda) \int_{a}^{x} d \mathfrak{F}(t, \lambda) \mathfrak{Y}(t, \lambda) \mathfrak{Y}\right)^{-1}(x, \lambda) .
$$

An equivalent form for the solution is

$$
\left.\mathfrak{z}(x, \lambda)=-\mathfrak{F}(\lambda) \int_{x}^{b} d \mathfrak{F}(t, \lambda) \mathfrak{Y}(t, \lambda) \mathfrak{Y}\right)^{-1}(x, \lambda) .
$$

The equivalence may be established by noting that the difference of the two forms is zero.

On the surface, the adjoint system appears to be nonhomogeneous. Nevertheless, its solutions have the characteristic linear properties of solutions of homogeneous systems. Thus, if $z(x, \lambda)$ is any solution with parametric vector $\mathfrak{f}(\lambda)$, it is easily verified that, for any scalar $c, c \mathfrak{z}(x, \lambda)$ is a solution with parametric vector $c \mathfrak{f}(\lambda)$. Also, if $z_{1}(x, \lambda)$ and $z_{2}(x, \lambda)$ are two solutions with parametric vectors $\mathfrak{f}_{1}(\lambda)$ and $\mathfrak{f}_{2}(\lambda)$, respectively, then $z_{1}(x, \lambda)+z_{2}(x, \lambda)$ is a solution with parametric vector $\mathfrak{f}_{1}(\lambda)+\mathfrak{f}_{2}(\lambda)$.

THEOREM 2. The given boundary system and its adjoint system have the same characteristic values and the same order of compatibility at each characteristic value.

Proof. The characteristic values for both systems are the values of $\lambda$ for which $\mathfrak{D}(\lambda)$ is singular. Also, if $\mathfrak{D}(\lambda)$ has rank $n-r$, there will be $r$ independent parametric vectors orthogonal to it, and the system will be compatible to the order $r$.

The following theorem lists the properties of Green's matrix relative to the adjoint system.

THEOREM 3. Green's matrix has the following additonal properties:

(1) For each fixed $x, \mathfrak{G}(x, s, \lambda)$ is a formal solution of system (4.1).

(2) If $\lambda$ is not a characteristic value, a solution of the equation

$$
\mathfrak{Z}^{\prime}=-\mathfrak{3} \mathfrak{X}(s, \lambda)+\mathfrak{A}(\lambda) \mathfrak{W}(s, \lambda)+\mathfrak{B}(s),
$$

with boundary conditions (4.1b) is given by

$$
\mathfrak{3}(s, \lambda)=-\int_{a}^{b} \mathfrak{B}(x) \mathfrak{G}(x, s, \lambda) d x .
$$


Proof. By comparing the top line of (3.1) with (4.4) and the bottom line with (4.5), we see that Green's matrix is a formal solution of $(4.1 \mathrm{a})$ with $\mathfrak{Y}(x, \lambda) \mathfrak{D}^{-1}(\lambda)$ as the parametric matrix. Its discontinuities, exhibited in (3.2), are precisely those required by (4.1b). Property (1) is therefore established.

Since $\mathfrak{G}(x, s, \lambda)$ is discontinuous at $x=s$, differentiation of (4.7) yields

$$
\mathfrak{Z}^{\prime}(s, \lambda)=-\int_{a}^{b} \mathfrak{B}(x) \frac{\partial}{\partial s} \mathfrak{G}(x, s, \lambda) d x-\mathfrak{B}(s)\left\{\mathfrak{G}\left(s^{-}, s, \lambda\right)-\mathfrak{G}\left(s^{+}, s, \lambda\right)\right\} .
$$

In view of property (1),

$$
\frac{\partial}{\partial s} \mathfrak{b}(x, s, \lambda)=-\mathfrak{G}(x, s, \lambda) \mathfrak{U}(s, \lambda)+\mathfrak{R}(\lambda) \mathfrak{W}(s, \lambda),
$$

where

$$
\mathfrak{R}(\lambda)=\mathfrak{Y}(x, \lambda) \mathfrak{D}^{-1}(\lambda) .
$$

Hence, recalling that $\mathfrak{G}\left(s^{+}, s, \lambda\right)-\mathfrak{G}\left(s^{-}, s, \lambda\right)=\mathfrak{J}$, we have

$$
\begin{aligned}
\mathfrak{Z}^{\prime}(s, \lambda) & =\int_{a}^{b} \mathfrak{B}(x) \mathfrak{b}(x, s, \lambda) d x \mathfrak{U}(s, \lambda)-\int_{a}^{b} \mathfrak{B}(x) \mathfrak{Y}(x, \lambda) \mathfrak{D}^{-1}(\lambda) d x \mathfrak{W}(s, \lambda)+\mathfrak{B}(s) \\
& =-\mathfrak{Z}(s, \lambda) \mathfrak{U}(s, \lambda)+\mathfrak{R}_{1}(\lambda) \mathfrak{W}(s, \lambda)+\mathfrak{B}(s),
\end{aligned}
$$

where

$$
\mathfrak{R}_{1}(\lambda)=-\int_{a}^{b} \mathfrak{B}(x) \mathfrak{Y}(x, \lambda) \mathfrak{D}^{-1}(\lambda) d x .
$$

The matrix $3(s, \lambda)$ is therefore seen to be a solution of the equation (4.6) with $\Omega_{1}(\lambda)$ as the parametric matrix.

The discontinuity of $3(s, \lambda)$ at $a_{h}$ is given by

$$
\begin{aligned}
\mathfrak{Z}\left(a_{h}^{+}, \lambda\right)-\mathfrak{Z}\left(a_{h}^{-}, \lambda\right) & =-\int_{a}^{b} \mathfrak{B}(x)\left\{\mathfrak{G}\left(x, a_{h}^{+}, \lambda\right)-\mathfrak{G}\left(x, a_{h}^{-}, \lambda\right)\right\} d x \\
& =-\int_{a}^{b} \mathfrak{B}(x) \mathfrak{Y}(x, \lambda) \mathfrak{D}^{-1}(\lambda) \mathfrak{W}^{(h)}(\lambda) d x \\
& =\mathfrak{R}_{1}(\lambda) \mathfrak{W}^{(h)}(\lambda), \quad h=1,2, \cdots, m .
\end{aligned}
$$

Hence, $3(s, \lambda)$ satisfies the boundary conditions $(4.1 \mathrm{~b})$, and the theorem is proved.

The relation established in the next theorem will be useful in $\$ \$ 11$ and 12 . It is, in essence, Green's formula for the system and its adjoint.

THEOREM 5. If $3(x, \lambda)$ is any solution of the adjoint system and $\mathfrak{B}(x)$ is any matrix with an integrable derivative, then 


$$
\int_{a}^{b} \mathfrak{Z}(x, \lambda)\left\{\mathfrak{B}^{\prime}(x)-\mathfrak{A}(x, \lambda) \mathfrak{B}(x)\right\} d x=-\mathfrak{R}(\lambda) \int_{a}^{b} d \mathfrak{F}(x, \lambda) \mathfrak{B}(x),
$$

where $\mathfrak{R}(\lambda)$ is a parametric matrix associated with $3(x, \lambda)$.

Proof. Since it is assumed that $3(x, \lambda)$ satisfies (4.1a),

$$
\frac{d}{d x}(\mathfrak{Z}(x, \lambda) \mathfrak{B}(x))=\mathfrak{Z}(x, \lambda)\left\{\mathfrak{B}^{\prime}(x)-\mathfrak{A}(x, \lambda) \mathfrak{B}(x)\right\}+\mathfrak{R}(\lambda) \mathfrak{W}(x, \lambda) \mathfrak{B}(x) .
$$

Integrating both sides of this relation and recalling that $3(x, \lambda)$ is discontinuous at the boundary points, we get

$$
\begin{aligned}
-\sum_{h=1}^{m}\left(\mathfrak{Z}\left(a_{h}^{+}, \lambda\right)-\mathfrak{Z}\left(a_{h}^{-}, \lambda\right)\right) \mathfrak{B}\left(a_{h}\right)= & \int_{a}^{b} \mathfrak{Z}(x, \lambda)\left\{\mathfrak{B}^{\prime}(x)-\mathfrak{U}(x, \lambda) \mathfrak{B}(x)\right\} d x \\
& +\mathfrak{H}(\lambda) \int_{a}^{b} \mathfrak{W}(x, \lambda) \mathfrak{B}(x) d x
\end{aligned}
$$

Since $3(x, \lambda)$ satisfies conditions (4.1b), relation (4.9) follows at once.

5. Remarks. When $\mathfrak{W}(x, \lambda) \equiv \mathfrak{D}$ and $m=2$, the system reduces to the classical 2-point boundary problem. The adjoint boundary relations, in this case, impose a parametrically defined linear condition on the values of the solution at the end points of the interval. The parametric form of the adjoint conditions proves to be more convenient and less restrictive than the nonparametric form. In particular, the familiar developments associated with an $n$th order linear equation and 2-point boundary conditions are greatly simplified by reducing the system to matrix form and applying the above results.

If $\mathfrak{M}(x, \lambda) \equiv \mathfrak{O}$ and $m$ is any finite positive integer, we have the $m$-point case [1]. The developments contained in $\$ \S 6$ and 7 , below, were indicated by the results for the $m$-point case. It is interesting to note that the use of the Stieltjes integral here provides a treatment of the general case which is less complicated than the treatment given in reference [1].

If $\mathfrak{M}(x, \lambda) \not \equiv \mathfrak{D}$ and $m=2$, we have the case discussed by Whyburn [7], with the advantage here that the Green's matrix is a formal solution of the adjoint conditions, and neither $\mathfrak{W}^{(1)}(\lambda)$ nor $\mathfrak{W}^{(2)}(\lambda)$ is required to have an inverse. The fact that interface conditions arise in the adjoint system is of interest, since such conditions have received a considerable amount of independent attention [4].

Finally, it is significant that the integrand of the integral part of the boundary relation of the given system appears in the adjoint equation and not in the adjoint boundary conditions. An intuitive appreciation of this phenomenon can be achieved by regarding the integral conditions as a limiting case of the $m$-point conditions. Thus, the condition

$$
\int_{a}^{b} d \mathfrak{F}(x, \lambda) \mathfrak{Y}(x, \lambda)=\mathfrak{D}
$$


is the discrete $m$-point condition, if $\mathfrak{F}(x, \lambda)=\mathfrak{F}_{1}(x, \lambda)$ as defined in $\$ 2$. The corresponding adjoint condition is

$$
3\left(a_{h}^{+}, \lambda\right)-\mathfrak{Z}\left(a_{h}^{-}, \lambda\right)=\mathfrak{R}(\lambda) \mathfrak{W}^{(h)}(\lambda),
$$

where $\mathfrak{B}^{(h)}(\lambda)$ is the saltus of $\mathfrak{F}(x, \lambda)$ at $a_{h}$. Clearly, the continuous counterpart of this, when $\mathfrak{F}(x, \lambda)$ is continuous and given by $(2.2)$, is

$$
\mathfrak{Z}^{\prime}(x, \lambda)=\mathfrak{R}(\lambda) \mathfrak{M}(x, \lambda) \text {. }
$$

The addition of $\mathfrak{R}(\lambda) \mathfrak{B}(x, \lambda)$ to the right side of the adjoint equation is thereby suggested.

Whyburn has pointed out [8] that the requirements of scientific research reveal a need for mathematical devices that will deal with an infinite number of interface points and "carry over a limit point (critical point) into a new reaction phase for the physical system." The interrelationships exhibited here may prove advantageous in this respect.

6. The reduction of Green's matrix. The reduction of Green's matrix, achieved in this section, is suggested by a similar reduction in the $m$-point case $[1, \S 4]$. Its derivation here parallels the derivation given in that case.

The characteristic matrix, defined in (2.6), may also be written in the form

$$
\mathfrak{D}(\lambda)=\int_{a}^{b} d \mathfrak{U}(x, \lambda)
$$

if the matrix $\mathfrak{U}(x, \lambda)$ is defined by

$$
\begin{aligned}
\mathfrak{U}^{\prime}(x, \lambda) & =\mathfrak{F}^{\prime}(x, \lambda) \mathfrak{Y}(x, \lambda), & & x \neq a_{h}, \\
\mathfrak{U}\left(a_{h}^{+}, \lambda\right)-\mathfrak{U}\left(a_{h}^{-}, \lambda\right) & =\mathfrak{W}^{(h)}(\lambda) \mathfrak{Y}\left(a_{h}, \lambda\right), & & h=1,2, \cdots, m .
\end{aligned}
$$

The latter relation is made complete with respect to the end points by specifying that

$$
\mathfrak{U}\left(a_{1}^{-}, \lambda\right)=\mathfrak{U}\left(a_{1}, \lambda\right)=\mathfrak{D} \text { and } \mathfrak{U}\left(a_{m}^{+}\right)=\mathfrak{U}\left(a_{m}\right) .
$$

Let the matrix $\overline{\mathfrak{G}}(x, s, \mu, \lambda)$ be defined by

$$
\overline{\mathfrak{G}}(x, s, \mu, \lambda)=\mathfrak{Y}(x, \lambda) \mathfrak{D}^{-1}(\lambda) \mathfrak{U}(\mu, \lambda) \mathfrak{Y}^{-1}(s, \lambda) .
$$

Since

$$
\int_{a}^{s} d \mathfrak{F}(t, \lambda) \mathfrak{Y}(t, \lambda)=\int_{a}^{s} d \mathfrak{U}(\mu, \lambda),
$$

we may replace the definition of $\mathfrak{G}(x, s, \lambda)$, given in (3.1), by 


$$
\mathfrak{G}(x, s, \lambda)=\left\{\begin{array}{rr}
\int_{a}^{s} d_{\mu} \overline{\mathfrak{F}}(x, s, \mu, \lambda), & s<x, \\
-\int_{s}^{b} d_{\mu} \overline{\mathfrak{F}}(x, s, \mu, \lambda), & s>x .
\end{array}\right.
$$

In this relation the subscript $\mu$ on the differential symbol indicates the variable of integration for the Stieltjes integral.

The following lemma is pertinent to the reduction of Green's matrix.

LEMMA 6.1. If $\mathfrak{U}(\mu)$ is an $n \times n$ matrix and if $\mathfrak{D}=\int_{a}^{b} d \mathfrak{U}(\mu)$ is nonsingular, there exist matrices $\mathfrak{S}_{1}(\mu)$ and $\mathfrak{S}_{2}(\mu, v)$ such that

with

$$
\mathfrak{D}^{-1} \mathfrak{U}(\mu)=\mathfrak{H}_{1}(\mu)+\int_{a}^{b} d_{v} \mathfrak{H}_{2}(\mu, v)
$$

$$
\mathfrak{H}_{2}(\mu, v)=-\mathfrak{H}_{2}(v, \mu) .
$$

The matrix $\mathfrak{H}_{1}(\mu)$ has zero components except on its diagonal where each component is the corresponding diagonal component of $\mathfrak{D}^{-1} \mathfrak{U}(\mu)$.

Proof. This lemma is the analog of Lemma 1 in the $m$-point case $[1, \S 4]$. The proof given there is adapted to the present case by replacing certain summations by integrations and, correspondingly, by changing indices of summation to variables of integration.

Let the symbol $\mathfrak{I}_{l l}$ represent the matrix in which all the components are zero except for a unit component in the $h$ th row and $l$ th column. Let the matrix $\mathfrak{I}^{\text {hh }}$ be defined by

$$
\mathfrak{J}^{h h}=\mathfrak{I}-\mathfrak{J}_{h h}
$$

If $D$ is the determinant of $\mathfrak{D}$, then

Hence,

$$
\mathfrak{D}^{-1}=1 / D \quad\left(\left|\mathfrak{D} \mathfrak{I}^{i i}+\mathfrak{I}_{j i}\right|\right) \text {. }
$$

$$
\begin{aligned}
\mathfrak{D}^{-1} \mathfrak{U}(\mu) & =1 / D \quad\left(\left|\mathfrak{D I}^{i i}+\mathfrak{U}(\mu) \mathfrak{I}_{j i}\right|\right) \\
& =\left(\frac{\delta_{i j}}{D}\left|\mathfrak{D} \mathfrak{I}^{i i}+\mathfrak{U}(\mu) \mathfrak{I}_{j i}\right|\right)+\left(\frac{1-\delta_{i j}}{D}\left|\mathfrak{D} \mathfrak{I}^{i i}+\mathfrak{U}(\mu) \mathfrak{I}_{j i}\right|\right) .
\end{aligned}
$$

Let the first matrix on the right be represented by $\mathfrak{H}_{1}(\mu)$. It is a diagonal matrix so that we may replace the index $i$ by $j$ and write

$$
\mathfrak{S}_{1}(\mu)=\left(\frac{\delta_{i j}}{D}\left|\mathfrak{D}^{j j}+\mathfrak{U}(\mu) \mathfrak{I}_{j j}\right|\right) \text {. }
$$

Each component of $\mathfrak{D}$ is the integral of the corresponding component of $\mathfrak{U}(\mu)$. 
In particular, we may so express the components of the $j$ th column of $\mathfrak{D}$ in the second term on the right of formula (6.4). That is,

$$
\mathfrak{D}^{-1} \mathfrak{U}(\mu)=\mathfrak{H}_{1}(\mu)+\left(\frac{1-\delta_{i j}}{D}\left|\mathfrak{D} \mathfrak{J}^{i i} \mathfrak{J}^{j j}+\int_{a}^{b} d_{v} \mathfrak{U}(v) \mathfrak{I}_{j j}+\mathfrak{U}(\mu) \mathfrak{I}_{j i}\right|\right)
$$

The use of $v$ as the variable of integration in the jth column of the determinant distinguishes that column. In consequence, since the expansion of the determinant may be expressed as a linear combination of the components of the $j$ th column, the integration symbol may be written outside the determinantal bar and thence outside the matrix bracket. Thus,

where

$$
\mathfrak{D}^{-1} \mathfrak{U}(\mu)=\mathfrak{H}_{1}(\mu)+\int_{a}^{b} d_{v} \mathfrak{H}_{2}(\mu, v),
$$

$$
\mathfrak{H}_{2}(\mu, v)=\left(\frac{1-\delta_{i j}}{\mathrm{D}}\left|\mathfrak{D} \mathfrak{J}^{i i} \mathfrak{I}^{j j}+\mathfrak{U}(v) \mathfrak{I}_{j j}+\mathfrak{U}(\mu) \mathfrak{I}_{j i}\right|\right) \text {. }
$$

Interchanging $\mu$ and $\nu$ in formula (6.6) has the effect of interchanging two columns in the determinant. Hence,

$$
\mathfrak{H}_{2}(\mu, v)=-\mathfrak{H}_{2}(v, \mu) \text {. }
$$

This proves the lemma.

If $c$ and $d$ are any two points on $[a, b]$, it is clear from (6.7) that

$$
\int_{c}^{d} d_{\mu} \int_{c}^{d} d_{v} \mathfrak{H}_{2}(\mu, v)=\mathfrak{D}
$$

Further, from (6.5),

$$
\int_{a}^{b} d_{\mu} \mathfrak{Y}_{1}(\mu)=\mathfrak{J}
$$

THEOREM 6. There exist matrices $\mathfrak{G}_{1}(x, s, \mu, \lambda)$ and $\mathfrak{G}_{2}(x, s, \mu, v, \lambda)$ such that

$$
\overline{\mathfrak{G}}(x, s, \mu, \lambda)=\mathfrak{G}_{1}(x, s, \mu, \lambda)+\int_{a}^{b} d_{v} \mathfrak{G}_{2}(x, s, \mu, v, \lambda),
$$

where

$$
\mathfrak{G}_{2}(x, s, \mu, v, \lambda)=-\mathfrak{G}_{2}(x, s, v, \mu, \lambda) .
$$

Proof. Let the matrix $\mathfrak{U}(\mu)$ of Lemma 6.1 be identified with the matrix $\mathfrak{U}(\mu, \lambda)$, appearing in formula (6.1). The matrix $\mathfrak{D}$ of the lemma becomes, then, the characteristic matrix $\mathfrak{D}(\lambda)$. Let $\mathfrak{G}_{1}(x, s, \mu, \lambda)$ and $\mathfrak{G}_{2}(x, s, \mu, v, \lambda)$ be defined by

$$
\begin{aligned}
\mathfrak{G}_{1}(x, s, \mu, \lambda) & =\mathfrak{Y}(x, \lambda) \mathfrak{H}_{1}(\mu) \mathfrak{Y}^{-1}(s, \lambda), \\
\mathfrak{F}_{2}(x, s, \mu, v, \lambda) & =\mathfrak{V}(x, \lambda) \mathfrak{H}_{2}(\mu, v) \mathfrak{Y}^{-1}(s, \lambda) .
\end{aligned}
$$


Hence, recalling (6.2) and Lemma 6.1, we obtain relation (6.8). Relation (6.9) follows from (6.7) and (6.11).

COROLlaRY. Employing relation (6.9), it may be inferred that

$$
\int_{c}^{d} d_{\mu} \int_{c}^{d} d_{v}\left(\mathfrak{G}_{2}(x, s, \mu, v, \lambda)=\mathfrak{D},\right.
$$

where $c$ and $d$ are any two points on $[a, b]$.

THEOREM 7. The formula for Green's matrix may be written as

$$
\mathfrak{G}(x, s, \lambda)=\left\{\begin{aligned}
\int_{a}^{s} d_{\mu}\left\{\mathfrak{G}_{1}(x, s, \mu, \lambda)+\int_{s}^{b} d_{v} \mathfrak{G}_{2}(x, s, \mu, v, \lambda)\right\}, & s<x, \\
-\int_{s}^{b} d_{\mu}\left\{\left(\mathfrak{G}_{1}(x, s, \mu, \lambda)+\int_{a}^{s} d_{v} \mathfrak{G}_{2}(x, s, \mu, v, \lambda)\right\},\right. & s>x .
\end{aligned}\right.
$$

Proof. Substituting from (6.8) in (6.3), we get

$$
\mathfrak{G}(x, s, \lambda)=\left\{\begin{aligned}
\int_{a}^{s} d_{\mu}\left\{\mathfrak{G}_{1}(x, s, \mu, \lambda)+\int_{a}^{b} d_{v}\left(\mathfrak{G}_{2}(x, s, \mu, v, \lambda)\right\},\right. & s<x, \\
-\int_{s}^{b} d_{\mu}\left\{\mathfrak{G}_{1}(x, s, \mu, \lambda)+\int_{a}^{b} d_{v}\left(\mathfrak{G}_{2}(x, s, \mu, v, \lambda)\right\},\right. & s>x .
\end{aligned}\right.
$$

By virtue of the corollary to Theorem 6 , with $c$ replaced by $a$ and $d$ by $s$, this may be reduced to the formula (6.12).

7. The structure of Green's matrix. In the ensuing discussion, it will be assumed that

$$
\mathfrak{U}(x, \lambda)=\lambda \mathfrak{R}(x)+\mathfrak{Q}(x),
$$

where $\mathfrak{R}(x)$ is the diagonal matrix $\left(\delta_{i j} r_{j}(x)\right)_{1}^{n}$, the diagonal components of the matrix $\mathfrak{Q}(x)$ are zeros, and the other components are indefinitely differentiable and free from $\lambda$. The functions $r_{j}(x), j=1,2, \cdots, n$, are indefinitely differentiable complex-valued functions which together with their differences, $r_{i}(x)-r_{j}(x), i \neq j$, have constant arguments and are bounded from zero for every $x$ on $[a, b]$.

Under these assumptions, Langer [2] has obtained the following asymptotic representation of a nonsingular solution of (2.1a).

$$
\mathfrak{Y}(x, \lambda)=\mathfrak{P}(x, \lambda) \mathfrak{E}(x, \lambda),
$$

where

$$
\mathfrak{E}(x, \lambda)=\left(\delta_{i j} e^{\lambda R_{j}(x)}\right)_{1}^{n}, \quad R_{j}(x)=\int_{a}^{x} r_{j}(t) d t,
$$

and $\mathfrak{P}(x, \lambda)$ has an asymptotic representation of the form 


$$
\mathfrak{P}(x, \lambda)=\mathfrak{T}+\sum_{h=1}^{k-1} \lambda^{-k} \mathfrak{P}^{(h)}(x)+\lambda^{-k} \mathfrak{B}_{k}(x, \lambda) \text {. }
$$

In the latter relation, $k$ is any natural number and $\mathfrak{P}^{(h)}(x), h=1,2, \cdots, k-1$, and $\mathfrak{B}_{k}(x, \lambda)$ are indefinitely differentiable in $x$, and $\mathfrak{B}_{k}(x, \lambda)$ is analytic in $\lambda$ and bounded for $|\lambda|$ large.

The matrix $\mathfrak{D}(\lambda)$ was represented in (6.1) in terms of the matrix $\mathfrak{U}(x, \lambda)$. It is convenient here to define the matrix $\mathfrak{B}(x, \lambda)$ by

$$
\begin{aligned}
\mathfrak{V}^{\prime}(x, \lambda) & =\mathfrak{W}(x, \lambda) \mathfrak{P}(x, \lambda), \quad x \neq a_{h}, \\
\mathfrak{B}\left(a_{h}^{+}, \lambda\right)-\mathfrak{B}\left(a_{h}^{-}, \lambda\right) & =\mathfrak{W}^{(h)}(\lambda) \mathfrak{P}\left(a_{h}, \lambda\right), \quad h=1,2, \cdots, m,
\end{aligned}
$$

where

$$
\mathfrak{B}\left(a_{1}^{-}, \lambda\right)=\mathfrak{B}\left(a_{1}, \lambda\right)=\mathfrak{D} \text { and } \mathfrak{B}\left(a_{m}^{+}, \lambda\right)=\mathfrak{B}\left(a_{m}, \lambda\right) .
$$

In view of this definition,

$$
\mathfrak{D}(\lambda) \mathrm{]}=\int_{a}^{b} d \mathfrak{B}(x, \lambda) \mathfrak{E}(x, \lambda) .
$$

Each component of $\mathfrak{D}(\lambda)$ is an integral, and its value is independent of the symbol used for the variable of integration. Consequently, we may write

$$
\mathfrak{D}(\lambda)=\left(\int_{a}^{b} e^{\lambda R_{j}\left(t_{j}\right)} d v_{i j}\left(t_{j}, \lambda\right)\right)_{1}^{n},
$$

so that each column of $\mathfrak{D}(\lambda)$ has a distinctive variable of integration.

The determinant $D(\lambda)$ of the matrix $\mathfrak{D}(\lambda)$ may be expressed as a sum in which each term is the product of $n$ integrals, one from each column of $\mathfrak{D}(\lambda)$. That is,

$$
D(\lambda)=\sum_{\left\{k_{\beta}\right\}} \int_{t_{n}=a}^{b} \cdots \int_{t_{1}=a}^{b} \exp \left\{\lambda \sum_{\alpha=1}^{n} R_{\alpha}\left(t_{\alpha}\right)\right\} d v_{k_{1} 1}\left(t_{1}, \lambda\right) \cdots d v_{k_{n} n}\left(t_{n}, \lambda\right),
$$

the summation applying to all permutations of the integers $1,2, \cdots, n$, for the set $\left\{k_{\beta} \mid \beta=1,2, \cdots, n\right\}$. The general term on the right side of (7.5) has been exhibited as an iterated integral and can be so written because of the use of a distinct variable of integration for each column of $\mathfrak{D}(\lambda)$. Relation (7.5) may be represented by

$$
D(\lambda)=\int_{S} \exp \left\{\lambda \sum_{\alpha=1}^{n} R_{\alpha}\left(t_{\alpha}\right)\right\}|d \mathfrak{V}|
$$

where $S$ is the $n$-dimensional region

$$
a \leqq t_{j} \leqq b, \quad j=1,2, \cdots, n .
$$

The unconventional symbol on the right side of (7.6) is to be regarded only as 
a convenient abbreviation for the right side of (7.5). The use of a similar abbreviation below will facilitate the comparison of the expressions so represented.

The integration of the matrix $\mathfrak{F}_{1}(x, s, \mu, \lambda)$ with respect to the variable $\mu$ is effected, in view of (6.10), by integrating $\mathfrak{S}_{1}(\mu)$. Recalling (6.5) and the equivalence of the right sides of (6.1) and (7.3), we may write

$$
\int_{\mu_{1}}^{\mu_{2}} d \mathfrak{S}_{1}(\mu)=\left(\frac{\delta_{i j}}{D}\left|\mathfrak{D} \mathfrak{I}^{j j}+\int_{\mu_{1}}^{\mu_{2}} d \mathfrak{B}(\mu, \lambda) \mathfrak{E}(\mu, \lambda) \mathfrak{I}_{j j}\right|\right) \text {. }
$$

The matrix appearing between the determinantal bars on the right side of this formula differs from the characteristic matrix only in respect to its $j$ th column. Consequently, the determinant admits of a symbolic representation similar to that given for $D(\lambda)$ in (7.6). That is,

$$
\int_{\mu_{1}}^{\mu_{2}} d \mathfrak{S}_{1}(\mu)=\left(\frac{\delta_{i j}}{D} \int_{S_{j}} \exp \left\{\lambda\left[R_{j}(\mu)+\sum_{\alpha=1, \alpha \neq j}^{n} R_{\alpha}\left(t_{\alpha}\right)\right]\right\}\left|d \mathfrak{B}_{j}\right|\right),
$$

where $S_{j}$ is an abbreviation for $S_{j}\left(\left[\mu_{1}, \mu_{2}\right]\right)$ and represents the $n$-dimensional region

$$
\begin{aligned}
& a \leqq t_{\alpha} \leqq b, \quad \alpha=1,2, \cdots, n, \quad \alpha \neq j, \\
& \mu_{1} \leqq \mu \leqq \mu_{2},
\end{aligned}
$$

and $\mathfrak{B}_{j}$ is the matrix $\mathfrak{B}$ used in (7.6) with its $j$ th column replaced by the $j$ th column of $\mathfrak{B}(\mu, \lambda)$.

When $\mathfrak{S}_{2}(\mu, v)$ is integrated with respect to its two variables, we obtain a matrix whose general term lends itself to the same type of symbolic representation. Thus, using (6.6), we have

$$
\begin{aligned}
\int_{\mu=\mu_{1}}^{\mu_{2}} d_{\mu} & \int_{v=v_{1}}^{v_{2}} d_{v \mathfrak{S}_{2}}(\mu, v) \\
& =\left(\frac{1-\delta_{i j}}{D} \int_{S_{i j}} \exp \left\{\lambda\left[R_{j}(\mu)+R_{j}(v)+\sum_{\alpha=1, \alpha \neq i, j}^{n} R_{\alpha}\left(t_{\alpha}\right)\right]\right\}\left|d \mathfrak{Q}_{i j}\right|\right),
\end{aligned}
$$

where $S_{i j}$ is an abbreviation for $S_{i j}\left(\left[\mu_{1}, \mu_{2}\right],\left[v_{1}, v_{2}\right]\right)$ and represents the $n$ dimensional region

$$
\begin{aligned}
& a \leqq t_{\alpha} \leqq b, \\
& \mu_{1} \leqq \mu \leqq \mu_{2}, \alpha=1,2, \cdots, n, \quad \alpha \neq i, j, \\
& v_{1} \leqq v \leqq v_{2} .
\end{aligned}
$$

$\mathfrak{B}_{i j}$ is the matrix $\mathfrak{B}$ used in (7.6) with its $i$ th column replaced by the $j$ th column of $\mathfrak{B}(\mu, \lambda)$ and its $j$ th column replaced by the $j$ th column of $\mathfrak{B}(v, \lambda)$.

Using (6.10), we get

$$
\int_{\mu_{1}}^{\mu_{2}} d_{\mu} \mathfrak{G}_{1}(x, s, \mu, \lambda)=\mathfrak{P}(x, \lambda) \mathfrak{E}(x, \lambda) \int_{\mu_{1}}^{\mu_{2}} d \mathfrak{S}_{1}(\mu) \mathfrak{E}^{-1}(s, \lambda) \mathfrak{P}^{-1}(s, \lambda) .
$$

Since 


$$
\mathbb{E}^{-1}(s, \lambda)=\left(\delta_{i j} e^{-\lambda R_{j}(s)}\right),
$$

if $\phi_{j}(x, s)$ is defined by

$$
\phi_{j}(x, s)=R_{j}(x)-R_{j}(s)+R_{j}(\mu)+\sum_{\alpha=1, \alpha \neq j}^{n} R_{\alpha}\left(t_{\alpha}\right),
$$

we may write

$$
\int_{\mu_{1}}^{\mu_{2}} d_{\mu}\left(\mathfrak{G}_{1}(x, s, \mu, \lambda)=\left(\sum_{j=1}^{n} p_{r j}(x, \lambda) p^{j c}(s, \lambda) \int_{S_{j}} \frac{1}{D} \exp \left\{\lambda \phi_{j}(x, s)\right\}\left|d \mathfrak{B}_{j}\right|\right)_{r, c=1}^{n},\right.
$$

where $\mathfrak{P}(x, \lambda)=\left(p_{r c}(x, \lambda)\right)_{1}^{n}$ and $\mathfrak{P}^{-1}(s, \lambda)=\left(p^{r c}(s, \lambda)\right)_{1}^{n}$. The components of $\mathfrak{P}^{-1}(s, \lambda)$ are readily seen to be polynomials in $1 / \lambda$ with coefficients which are indefinitely differentiable functions of $s$.

In a similar fashion, with the definition

$$
\phi_{i j}(x, s)=R_{i}(x)-R_{j}(s)+R_{j}(\mu)+R_{j}(v)+\sum_{\alpha=1, \alpha \neq i, j}^{n} R_{\alpha}\left(t_{\alpha}\right),
$$

the integration of (6.11) yields

$$
\begin{aligned}
& \int_{\mu_{1}}^{\mu_{2}} d_{\mu} \int_{v_{1}}^{v_{2}} d_{v}\left(\mathfrak{G}_{2}(x, s, \mu, v, \lambda)\right. \\
& \quad=\left(\sum_{i, j=1, i \neq j}^{n} p_{r i}(x, \lambda) p^{j c}(s, \lambda) \int_{s_{i j}} \frac{1}{D} \exp \left\{\lambda \phi_{i j}(x, s)\right\}\left|d \mathfrak{B}_{i j}\right|\right)_{r, c=1}^{n} .
\end{aligned}
$$

The matrix $\mathfrak{F}(x, s, \lambda)$, as expressed in (6.12), is seen to be the sum of the matrices given by formulas (7.8) and (7.10), if appropriate limits of integration are specified. Thus, each component of $\mathfrak{G}(x, s, \lambda)$ is $1 / D$ times a linear combination of determinants. Each determinant in the linear combination is similar in form to the determinant $D$. The nature of the denominator determinant, $D$, is clearly significant for the ensuing discussion.

8. The chacteristic values. For the discussion of the formal aspects of the system it was convenient to combine the discrete and continuous parts of the boundary conditions by means of a Stieltjes integral. It is desirable here to separate these parts and write

$$
\mathfrak{D}(\lambda)=\mathfrak{D}_{1}(\lambda)+\mathfrak{D}_{2}(\lambda),
$$

where

$$
\begin{aligned}
& \mathfrak{D}_{1}(\lambda)=\sum_{h=1}^{m} \mathfrak{W}^{(h)}(\lambda) \mathfrak{Y}\left(a_{h}, \lambda\right), \\
& \mathfrak{D}_{2}(\lambda)=\int_{a}^{b} \mathfrak{W}(x, \lambda) \mathfrak{Y}(x, \lambda) d x
\end{aligned}
$$


If $\sigma$ is the degree of the highest degree polynomial appearing as a component of any of the matrices $\mathfrak{W}^{(h)}(\lambda), h=1,2, \cdots, m$, and $\mathfrak{W}(x, \lambda)$, we may define $\mathfrak{U}^{*(h)}(\lambda)$ and $\mathfrak{U}^{*}(x, \lambda)$ by

$$
\begin{aligned}
\lambda^{\sigma} \mathfrak{U}^{*(h)}(\lambda) & =\mathfrak{B}^{(h)}(\lambda) \mathfrak{P}\left(a_{h}, \lambda\right), \quad h=1,2, \cdots, m, \\
\lambda^{\sigma} \mathfrak{U}^{*}(x, \lambda) & =\mathfrak{M}(x, \lambda) \mathfrak{P}(x, \lambda) .
\end{aligned}
$$

(The starred symbols are used here to avoid possible confusion with the symbols used in §6.) When defined as above, the components of $\mathfrak{U}^{*(h)}(\lambda)$ and $\mathfrak{I}^{*}(x, \lambda)$ are asymptotic polynomials in $1 / \lambda$.

In view of (8.1),

$$
\mathfrak{D}_{1}(\lambda)=\lambda^{\sigma} \sum_{h=1}^{m} \mathfrak{U}^{*(h)}(\lambda) \mathfrak{E}\left(a_{h}, \lambda\right),
$$

and the general component of $\mathfrak{D}_{1}(\lambda)$ is

$$
d_{1, i j}(\lambda)=\lambda^{\sigma} \sum_{h=1}^{m} u_{i j}^{(h)}(\lambda) e^{\lambda R\left(a_{h}\right)} .
$$

Using (8.2), we get

$$
\mathfrak{D}_{2}(\lambda)=\lambda^{\sigma} \int_{a}^{b} \mathfrak{U}^{*}(x, \lambda) \mathfrak{E}(x, \lambda) d x .
$$

If this is written as

$$
\mathfrak{D}_{2}(\lambda)=\lambda^{\sigma-1} \int_{a}^{b} \mathfrak{U}^{*}(x, \lambda) \Re^{-1}(x) \lambda \mathfrak{R}(x) \mathfrak{E}(x, \lambda) d x,
$$

and $\mathfrak{U}_{1}(x, \lambda)$ is defined by

$$
\mathfrak{U}_{1}(x, \lambda)=-\frac{d}{d x}\left\{\mathfrak{U}^{*}(x, \lambda) \Re^{-1}(x)\right\},
$$

integration by parts leads to

$$
\mathfrak{D}_{2}(\lambda)=\lambda^{\sigma-1}\left[\mathfrak{U}^{*}(b, \lambda) \Re^{-1}(b) \mathfrak{E}(b, \lambda)-\mathfrak{U}^{*}(a, \lambda) \mathfrak{R}^{-1}(a) \mathfrak{E}(a, \lambda)+\int_{a}^{b} \mathfrak{U}_{1}(x, \lambda) \mathfrak{E}(x, \lambda) d x\right] .
$$

Repeated integration by parts, where

$$
\begin{aligned}
\mathfrak{U}_{l}(x, \lambda) & =-\frac{d}{d x}\left\{\mathfrak{U}_{l-1}(x, \lambda) \mathfrak{R}^{-1}(x)\right\}, \quad l=1,2, \cdots, \\
\mathfrak{U}_{0}(x, \lambda) & \equiv \mathfrak{U}^{*}(x, \lambda),
\end{aligned}
$$

yields 
$\mathfrak{D}_{2}(\lambda)=\lambda^{\sigma-1}\left[\sum_{l=0}^{p} \lambda^{-l}\left\{\mathfrak{U}_{l}(b, \lambda) \mathfrak{R}^{-1}(b) \mathfrak{E}(b, \lambda)-\mathfrak{U}_{l}(a, \lambda) \mathfrak{R}^{-1}(a) \mathfrak{E}(a, \lambda)\right\}\right.$

$$
\left.+\lambda^{-p} \int_{a}^{b} \mathfrak{U}_{p+1}(x, \lambda) \mathfrak{E}(x, \lambda) d x\right] .
$$

where $p$ is any non-negative integer. If $\mathfrak{A}(\lambda)$ and $\mathfrak{B}(\lambda)$ are defined by

$$
\begin{aligned}
& \mathfrak{U}(\lambda)=-\sum_{l=0}^{p} \lambda^{-l} \mathfrak{U}_{l}(a, \lambda) \mathfrak{R}^{-1}(a), \\
& \mathfrak{B}(\lambda)=\sum_{l=0}^{p} \lambda^{-l} \mathfrak{U}_{l}(b, \lambda) \mathfrak{R}^{-1}(b),
\end{aligned}
$$

formula (8.4) can be written as

$$
\mathfrak{D}_{2}(\lambda)=\lambda^{\sigma-1}\left[\mathfrak{A}(\lambda) \mathfrak{E}(a, \lambda)+\mathfrak{B}(\lambda) \mathfrak{E}(b, \lambda)+\lambda^{-p} \int_{a}^{b} \mathfrak{U}_{p+1}(x, \lambda) \mathfrak{E}(x, \lambda) d x\right] .
$$

The general component of $\mathfrak{D}_{2}(\lambda)$ is therefore given by

$$
d_{2, i j}(\lambda)=\lambda^{\nu-1}\left[a_{i j}(\lambda) e^{\lambda R_{j}(a)}+b_{i j}(\lambda) e^{\lambda R_{j}(b)}+\lambda^{-p} \int_{a}^{b} u_{p+1, i j}(x, \lambda) e^{\lambda R_{j}(x)} d x\right]
$$

where $a_{i j}(\lambda)$ and $b_{i j}(\lambda)$ are asymptotic polynomials in $1 / \lambda$, and $u_{p+1, i j}(\lambda)$ is analytic in $\lambda$ and bounded when $|\lambda|$ is large.

From the hypothesis that $r_{j}(x)$ has a constant argument, it follows that $R_{j}(x)$ has a constant argument. Since $r_{j}(x) \neq 0$ on $[a, b]$, the relation

$$
z=R_{j}(x), a \leqq x \leqq b,
$$

effects a one-to-one mapping of the interval $[a, b]$ onto the straight line segment in the $z$-plane joining the points $R_{j}(a)$ and $R_{j}(b)$. Clearly then, for any value of $\lambda$, at least one of the following relations remains valid for every $x$ on $[a, b]$. $(\operatorname{Re}\{z\}$ denotes the real part of $z$.)

$$
\begin{aligned}
& \operatorname{Re}\left\{\lambda\left(R_{j}(x)-R_{j}(a)\right)\right\} \leqq 0, \\
& \operatorname{Re}\left\{\lambda\left(R_{j}(x)-R_{j}(b)\right)\right\} \leqq 0 .
\end{aligned}
$$

If $\lambda$ is restricted to the remote part of the half-plane defined by (8.7), we may write

$$
d_{2, i j}(\lambda)=\lambda^{\sigma-1}\left[a_{i j}(\lambda) e^{\lambda R_{J}(a)}+b_{i j}(\lambda) e^{\lambda R_{j}(b)}+\lambda^{-p} g_{a}(\lambda) e^{\lambda R_{j}(a)}\right],
$$

where

$$
g_{a}(\lambda)=\int_{a}^{b} u_{p+1, i j}(x, \lambda) e^{\lambda\left(R_{f}(x)-R_{f}(a)\right)} d x
$$


Since $g_{a}(\lambda)$ is evidently analytic and bounded when $|\lambda|$ is large, the term in which it appears in formula (8.9) may be absorbed by the first term on the right side of the same formula without affecting the definition of that term. Similarly, if $\lambda$ is restricted to the remote part of the half-plane defined by (8.8), the integral term will be absorbed by the second term on the right side of formula (8.9). Thus, for $|\lambda|$ large,

$$
d_{2, i j}(\lambda)=\lambda^{\sigma-1}\left[a_{i j}(\lambda) e^{\lambda R_{J}(a)}+b_{i j}(\lambda) e^{\lambda R_{J}(b)}\right] .
$$

The general term of $\mathfrak{D}(\lambda)$ is obtained by adding (8.3) and (8.10). This results in an exponential sum which is similar in form to the right side of (8.3). Therefore, in general, the addition of $\mathfrak{D}_{2}(\lambda)$ to $\mathfrak{D}_{1}(\lambda)$ does not change the essential character of $\mathfrak{D}_{1}(\lambda)$ for large values of $|\lambda|$. Some consideration, however, must be given to the case where $a_{i j}(\lambda)$ (or $b_{i j}(\lambda)$ ) is asymptotically equal to zero. If, in this event, the coefficient $u_{i j}^{(1)}(\lambda)$ in (8.3) is not zero, there is no complication. On the other hand, if $u_{i j}^{(1)}(\lambda)=0$ and if $w_{i j}(x, \lambda) \equiv 0, a \leqq x \leqq c$, the formula (8.10) will be replaced by

$$
d_{2, i j}(\lambda)=\lambda^{\sigma-1}\left[c_{i j}(\lambda) e^{\lambda R_{j}(c)}+b_{i j}(\lambda) e^{\lambda R_{j}(b)}\right] .
$$

If $c$ is not one of the interior boundary points, the addition of this evaluation of $d_{2, i j}(\lambda)$ to $d_{1, i j}(\lambda)$ introduces a term into the latter exponential sum distinct from any originally present. In order to include the above situation with a minimum of notational effort, we shall restrict its occurrence to cases where the point $c$ coincides with one of the interior boundary points. This restriction can, in fact, be made without loss of generality, since such points can be introduced into the originally given set of interior boundary points and assigned zero coefficients in the boundary relation (2.1b). The general component in the matrix $\mathfrak{D}(\lambda)$, therefore, has the same form as the right side of (8.3). Thus, $\mathfrak{D}(\lambda)$ has the same form as the characteristic matrix arising in the case of discrete boundary relations $[1 ; 2]$.

The determinant of $\mathfrak{D}(\lambda)$ is the characteristic function. It is an exponential sum given by $[2,(11.3)]$

$$
D(\lambda)=\sum_{\alpha} A_{\alpha}(\lambda) e^{\lambda \Omega_{\alpha}},
$$

where $A_{\alpha}(\lambda)$ is an asymptotic polynomial in $1 / \lambda$ multiplied by a non-negative power of $\lambda$. The set of exponential coefficients,

is a subset of the set,

$$
E_{D}=\left\{\Omega_{\alpha} \mid A_{\alpha}(\lambda) \not \equiv 0\right\},
$$

$$
E=\left\{\sum_{\alpha=1}^{n} R\left(a_{k_{\alpha}}\right)\right\},
$$

where each member of the index set $k_{1}, k_{2}, \cdots, k_{n}$, is chosen independently from 
the integers $1,2, \cdots, m[2$, p. 173]. The elements of $E$ are complex numbers and may be plotted on a complex $z$-plane. Let $P$ represent the closed convex polygonal region of minimum area which contains all the points of $E$, and let $P_{D}$ be similarly defined relative to the points of the subset $E_{D}$.

The characteristic values of the boundary problem are the zeros of $D(\lambda)$. Their location and distribution have been described by Langer [2, $\$ 11,12]$. Briefly, they have no finite limit point and may be enumerated to form a sequence $\left\{\lambda_{j} \mid j=1,2, \cdots\right\}$ for which $\left|\lambda_{j}\right| \leqq\left|\lambda_{j+1}\right|$. A corresponding sequence of closed contours $\left\{\Gamma_{k}\right\}$ can be drawn in the $\lambda$-plane so that $\Gamma_{k}$ encloses precisely the first $k$ characteristic values, the index sequence $\{k\}$ is a nondecreasing sequence of positive integers, each contour encloses the origin and its predecessor in the sequence, and the shortest distance from the origin to $\Gamma_{k}$ becomes large with increasing values of $k$. As in $[2, \S 11]$, let the coefficient $A_{\alpha}(\lambda)$ in (8.11) be given the representation

$$
A_{\alpha}(\lambda)=\lambda^{\rho_{\alpha}} \sum_{k=0}^{\infty} A_{\alpha k} \lambda^{-k}
$$

where $A_{\alpha 0} \neq 0$ for each $\alpha$. Further, let the non-negative integer $\rho$ be the smallest integer in the set $\left\{\rho_{\alpha}\right\}$. The significant property of $D(\lambda)$ in relation to the set of contours is that the exponential sum

$$
\lambda^{-\rho} D(\lambda) e^{-\lambda \Omega_{\alpha}},
$$

where $\Omega_{\alpha}$ is any vertex of $P_{D}$, is bounded uniformly from zero if $\lambda$ is restricted to the contours of the set $\left\{\Gamma_{k}\right\}$.

9. Regularity of the boundary problem. In all discussions of expansion problems, regularity conditions are imposed to exclude what may be called irregular situations. Roughly speaking, it is desirable to exclude cases where the components of Green's matrix may become exponentially large in the remote part of the $\lambda$-plane. The form of the components of Green's matrix is effectively revealed in formulas (7.8) and (7.10). All the components are fractions with the characteristic function $D$ as a common denominator. The function $D$ was analyzed in $\$ 8$ and shown to be asymptotically equivalent to an exponential sum. The numerator of each fraction is an integral of an exponential sum. The relationship between the exponents in the numerator and those in the denominator clearly determines the nature of the fraction. The regularity conditions are designed to specify what may be called a satisfactory relationship between the exponents of the numerator and those of the denominator.

The functions $\phi_{j}(x, s)$ and $\phi_{i j}(x, s)$, defined by (7.7) and (7.9), will be represented here by $\phi_{j}\left(x, s, \mu ; t_{\alpha} \mid \alpha \neq j\right)$ and $\phi_{i j}\left(x, s, \mu, v ; t_{\alpha} \mid \alpha \neq i, j\right)$, respectively. The new notation, in the case of $\phi_{j}(x, s)$, is designed to indicate explicitly the 
dependence of the function on the parameters $\mu, t_{1}, t_{2}, \cdots, t_{j-1}, t_{j+1}, \cdots, t_{n}$. A similar remark applies to $\phi_{i j}(x, s)$. For each value of $x$ and $s$, the relation

$$
z=\phi_{j}\left(x, s, \mu ; t_{\alpha} \mid \alpha \neq j\right), \quad j=1,2, \cdots, n,
$$

may be used to map $S_{j}\left(\left[\mu_{1}, \mu_{2}\right]\right)$, the region of integration in formula (7.8), into a complex $z$-plane on which the polygonal regions $P$ and $P_{D}$ (defined in $\S 8$ ) have been indicated. Similarly,

$$
z=\phi_{i j}\left(x, s, \mu, v ; t_{\alpha} \mid \alpha \neq i, j\right), \quad i, j,=1,2, \cdots, n, i \neq j,
$$

will map $S_{i j}\left(\left[\mu_{1}, \mu_{2}\right],\left[v_{1}, v_{2}\right]\right)$ into the same $z$-plane.

Definition. The boundary problem will be said to be regular relative to a specific value of $x$ if

(1) for every $s$ on $[a, x)$, the images of $S_{j}([a, s])$ under (9.1) and of $S_{i j}([a, s],[s, b])$ under $(9.2)$ are both in $P_{D}$, and

(2) for every $s$ on $(x, b]$, the images of $S_{j}([s, b])$ under (9.1) and of $S_{i j}([s, b],[a, s])$ under $(9.2)$ are both in $P_{D}$.

The boundary problem will be said to be regular relative to any subinterval of $[a, b]$ if it is regular relative to every $x$ on that subinterval.

The regions of the parameter space, mentioned in the definition of regularity, are precisely the regions of integration determined by substituting from relations (7.8) and (7.10) in (6.12). It is therefore clear that if the regularity conditions are satisfied, every exponent coefficient ( $\phi$-function) appearing in $\mathfrak{G}(x, s, \lambda)$ will have values lying in $P_{D}$ for all values of the variable $s$.

The following theorem provides a sufficient condition for regularity.

THEOREM 8. If $P_{D}$ coincides with $P$, the boundary problem is regular.

Proof. From the one-to-one mapping property of relation (8.6) and the definition of $P$, it can be inferred that the relation

$$
z=\sum_{\alpha=1}^{n} R_{\alpha}\left(t_{\alpha}\right)
$$

maps the $n$-dimensional region, $a \leqq t_{\alpha} \leqq b, \alpha=1,2, \cdots, n$, onto the region $P$ and that the vertices of $P$ form a subset of the points obtained when each $t_{\alpha}$ in (9.3) is given independently one of the values $a$ or $b$.

If each variable and parameter, except one, in $\phi_{j}\left(x, s, \mu ; t_{\alpha} \mid \alpha \neq j\right)$ is held fast, any interval containing the free variable or parameter is mapped, one-to-one, into a straight line in the $z$-plane. Recalling (7.7), we see that $\phi_{j}\left(x, x, \mu ; t_{\alpha} \mid \alpha \neq j\right)$ and $\phi_{j}\left(x, \mu, \mu ; t_{\alpha} \mid \alpha \neq j\right)$ are both points in $P$. Hence, since $P$ is convex, $\phi_{j}\left(x, s, \mu ; t_{\alpha} \mid \alpha \neq j\right)$ is a point in $P$ for any $s$ between $x$ and $\mu$. It is merely a rephrasing of the latter statement to say that the point is in $P$ if $\mu$ is on $[a, s]$ when $s<x$ and on $[s, b]$ when $s>x$. Therefore, under (9.1), the image of $S_{j}([a, s])$ is in $P$ if $s<x$, and the image of $S_{j}([s, b])$ is in $P$ if $s>x$. 
Similarly, for the function $\phi_{i j}\left(x, s, \mu, v ; t_{\alpha} \mid \alpha \neq i, j\right)$, we infer from (7.9) that the points $\phi_{i j}\left(x, \mu, \mu, v ; t_{\alpha} \mid \alpha \neq i, j\right)$ and $\phi_{i j}\left(x, v, \mu, v ; t_{\alpha} \mid \alpha \neq i, j\right)$ are in $P$. Hence the point $\phi_{i j}\left(x, s, \mu, v ; t_{\alpha} \mid \alpha \neq i, j\right)$ is in $P$ for any $s$ between $\mu$ and $v$. That is, the point is in $P$ if one of the two parameters, $\mu$ and $v$, is on $[a, s]$ and the other on $[s, b]$. This is equivalent to saying that, under (9.2), the images of $S_{i j}([a, s],[s, b])$ and $S_{i j}([s, b],[a, s])$ are both in $P$. The theorem follows by comparing these results with the definition of regularity.

COROLLARY. Let $x$ be restricted to any closed interval which is in the interior of $[a, b]$ and on which the boundary problem is regular. The images of $S_{j}([a, s])$ and $S_{j}([s, b])$, under (9.1), are bounded away from the vertices of $P_{D}$ for $s$ bounded away from the end points of $[a, x)$ and $(x, b]$, respectively. Also, the images of $S_{i j}([a, s],[s, b])$ and $S_{i j}([s, b],[a, s])$, under $(9.2)$, are bounded away from the vertices of $P_{D}$ for $s$ bounded away from the end points of $[a, x)$ and $(x, b]$, respectively.

This result follows from the fact that $R_{j}^{\prime}(t) \neq 0, a \leqq t \leqq b$.

10. Residues of Green's matrix. Since the matrix $\mathfrak{D}(\lambda)$ is analytic, its inverse is analytic except for poles at the characteristic values. As noted by Langer [2], $\mathfrak{D}(\lambda)$ is not unique. It may, by a suitable choice of $\mathfrak{Y}(x, \lambda)$ and a nonsingular transformation of the boundary relation $(2.1 \mathrm{~b})$, be so adjusted that $[2,(13.1)]$

$$
\mathfrak{D}(\lambda)=\mathfrak{P}(\lambda)+\left(\lambda-\lambda_{\beta}\right)^{s+1} \mathfrak{D}^{(1)}(\lambda),
$$

where

(i) the integer $s$ is the multiplicity of the characteristic value $\lambda_{\beta}$,

(ii) $\mathfrak{D}^{(1)}(\lambda)$ is analytic at $\lambda=\lambda_{\beta}$,

(iii) $\mathfrak{P}(\lambda)=\left(\delta_{i j} p_{i}(\lambda)\right)$, with each $p_{i}(\lambda)$ a polynomial in $\left(\lambda-\lambda_{\beta}\right)$, and

$$
p_{i}\left(\lambda_{\beta}\right)=\left\{\begin{array}{l}
1, \text { for } i \leqq n-r, \\
0, \text { for } i>n-r .
\end{array}\right.
$$

Let $D_{j}$ be defined as the vector with 1 in the $j$ th position and zeros elsewhere. In general, it has not been necessary to make a notational distinction between row and column vectors, but it will be convenient below to use $\grave{D}_{j}$ only for a column vector and to represent the corresponding row vector by $\delta_{j}^{t}$. It is clear that $\mathfrak{D}_{j}, j=n-r+1, \cdots, n$, is orthogonal to $\mathfrak{D}\left(\lambda_{\beta}\right)$ on the right. Consequently, each vector

$$
\mathfrak{y}^{(l \beta)}(x)=\mathfrak{Y}\left(x, \lambda_{\beta}\right) \mathfrak{b}_{n-r+l}, \quad l=1,2, \cdots, r,
$$

is a characteristic solution of (1.2), and the $r$ vectors are linearly independent. It is also clear that $-\delta_{j}^{t}, j=n-r+1, \cdots, n$, is orthogonal to $\mathfrak{D}\left(\lambda_{\beta}\right)$ on the left and is a suitable choice for the parametric vector $\mathfrak{f}\left(\lambda_{\beta}\right)$ when $\lambda=\lambda_{\beta}$. Thus, using (4.4), 


$$
\mathfrak{Z}^{(l \beta)}(x)=-\mathfrak{D}_{n-r+l}^{t} \int_{a}^{x} d \mathfrak{F}\left(t, \lambda_{\beta}\right) \mathfrak{Y}\left(t, \lambda_{\beta}\right) \mathfrak{Y}^{-1}\left(x, \lambda_{\beta}\right), \quad l=1,2, \cdots, r,
$$

is a set of $r$ linearly independent solutions of (4.1).

The discussion, at this point, will be restricted to a characteristic value $\lambda_{\beta}$ whose multiplicity and index are the same. The polynomials $p_{i}(\lambda), i=n-r+1, \cdots, n$, have then simple zeros at $\lambda_{\beta}$, and $\mathfrak{D}^{-1}(\lambda)$ has corresponding simple poles. It can be inferred that $[2,(14.3)]$

$$
\operatorname{res}_{\beta} \mathfrak{D}^{-1}=\sum_{l=n-r+1}^{n} \mathfrak{D}_{l} \mathfrak{D}_{l}^{t},
$$

where the symbol $\operatorname{res}_{\beta} \mathfrak{D}^{-1}$ represents the residue of $\mathfrak{D}^{-1}(\lambda)$ at $\lambda_{\beta}$.

Since the poles of $\mathfrak{H}(x, s, \lambda)$ are contributed by the factor $\mathfrak{D}^{-1}(\lambda)$, it follows that

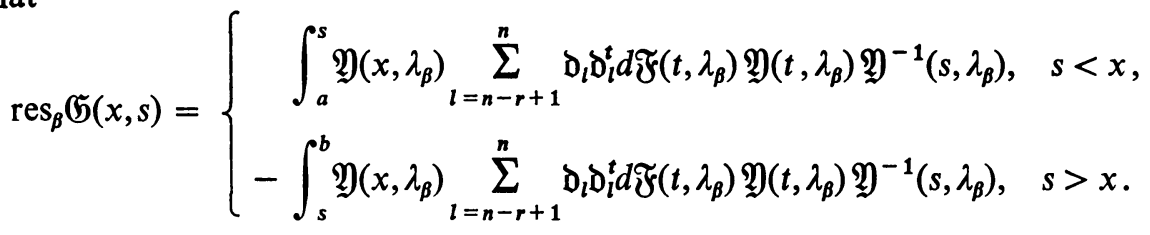

Hence, because of (10.1) and (10.2) and the equivalence of (4.4) and (4.5),

$$
\operatorname{res}_{\beta}\left(\mathfrak{G}(x, s)=-\sum_{l=1}^{r} \mathfrak{y}^{(l \beta)}(x) 3^{(l \beta)}(s)\right. \text {. }
$$

It should be noted that $z^{(l \beta)}(s)$ is a row vector so that $\mathfrak{y}^{(l \beta)}(x) 3^{(l \beta)}(s)$ is an $n \times n$ matrix. Also, since the matrix $(5(x, s, \lambda)$ is unique, the above result is independent of the adjustment made in the characteristic matrix. This adjustment served solely to facilitate the derivation of result.

11. The formal expansion. A familiar procedure for developing the expansion of a given vector is to determine orthogonality relations for the characteristic functions and to use these to determine the formal expansion of the vector. The terms of the expansion are then related to the residues of Green's matrix so that the partial sum of the series expansion can be represented by a contour integral. The behaviour of the integral, as the contour is enlarged to include more remote characteristic values, determines whether or not the series converges. This procedure could be followed in the present case, but it is more convenient to obtain the expansion by evaluating a contour integral. Orthogonality relations will be discussed briefly in $\$ 12$ in order to round out the discussion.

Let $\tau$ be a non-negative integer and let $f(s)$ be a vector with a bounded and integrable derivative of order $\tau+1$ on $[a, b]$. Consider the integral

where

$$
\frac{1}{2 \pi i} \int_{\Gamma_{k}} \mathfrak{h}(x, \lambda) d \lambda,
$$




$$
\mathfrak{h}(x, \lambda)=\int_{a}^{b} \mathfrak{G}(x, s, \lambda) \mathfrak{R}(s) \mathfrak{f}(s) d s .
$$

The consideration of this integral is motivated by the observation that it appears in most of the existing developments which can be regarded as special cases of the present development. In order to obtain an evaluation of $\mathfrak{h}(x, \lambda)$, let the variable $x$ in relation (4.9) be replaced by $s$ and let the matrix $\mathfrak{B}(s)$ be replaced by the vector $\mathfrak{f}(s)$. The matrix $\mathfrak{b}(x, s, \lambda)$, regarded as a function of $s$, is only a formal solution of (4.1). If, therefore, we replace $3(s, \lambda)$ in $(4.9)$ by $\mathfrak{b}(x, s, \lambda)$, the fact that $\mathfrak{G}\left(x, x^{+}, \lambda\right)-\mathfrak{G}\left(x, x^{-}, \lambda\right)=-\mathfrak{I}$ will introduce an additional term into the relation. The net result is

$$
\int_{a}^{b} \mathfrak{F}(x, s, \lambda)\left\{\mathfrak{f}^{\prime}(s)-\mathfrak{A}(s, \lambda) \mathfrak{f}(s)\right\} d s=\mathfrak{f}(x)-\mathfrak{R}(\lambda) \int_{a}^{b} d \mathfrak{F}(s, \lambda) \mathfrak{f}(s),
$$

where, in view of Theorem 3, the parametric matrix $\mathcal{R}(\lambda)$ is given by

$$
\mathfrak{R}(\lambda)=\mathfrak{V}(x, \lambda) \mathfrak{D}^{-1}(\lambda) .
$$

If $\mathfrak{A}(s, \lambda)$ is replaced by $\lambda \mathfrak{R}(s)+\mathfrak{Q}(s)$, relation (11.1) may be rewritten in the form

$$
\begin{aligned}
\int_{a}^{b} \mathfrak{G}(x, s, \lambda) \mathfrak{R}(s) \mathfrak{f}(s) d s= & -\frac{1}{\lambda} \mathfrak{f}(x)+\frac{1}{\lambda} \mathfrak{T}(\lambda) \int_{a}^{b} d \mathfrak{F}(s, \lambda) \mathfrak{f}(s) \\
& +\frac{1}{\lambda} \int_{a}^{b} \mathfrak{F}(x, s, \lambda) \Re(s) \mathfrak{f}^{(1)}(s) d s,
\end{aligned}
$$

where

$$
\mathfrak{f}^{(1)}(s)=\mathfrak{R}^{-1}(s)\left\{\mathfrak{f}^{\prime}(s)-\mathfrak{Q}(s) \mathfrak{f}(s)\right\} .
$$

The last term on the right of (11.3) is similar in form to the left side of the relation. By iteration then we get

$$
\begin{aligned}
\int_{a}^{b} \mathfrak{G}(x, s, \lambda) \mathfrak{R}(s) \mathfrak{f}(s) d s= & -\sum_{q=0}^{\tau} \lambda^{-q-1} \mathfrak{f}^{(q)}(x)+\mathfrak{R}(\lambda) \int_{a}^{b} d \mathfrak{F}(s, \lambda) \sum_{q=0}^{\tau} \lambda^{-q-1} \mathfrak{f}^{(q)}(s) \\
& +\lambda^{-\tau-1} \int_{a}^{b} \mathfrak{G}(x, s, \lambda) \mathfrak{R}(s) \mathfrak{f}^{(\tau+1)}(s) d s,
\end{aligned}
$$

where (see $[2,(15.3)])$

$$
\mathfrak{f}^{(0)}(s) \equiv \mathfrak{f}(s), \mathfrak{f}^{(q+1)}(s)=\mathfrak{R}^{-1}(s)\left\{\mathfrak{f}^{(q) \prime}(s)-\mathfrak{Q}(s) \mathfrak{f}^{(q)}(s)\right\}, \quad q=1,2, \cdots, \tau .
$$

Since

$$
\frac{1}{2 \pi i} \int_{\Gamma_{k}} \sum_{q=0}^{\tau} \lambda^{-q-1} \mathfrak{f}^{(q)}(x) d \lambda=\mathfrak{f}(x),
$$


where $\Gamma_{k}$ is the contour defined in $\S 8$, it follows that

$$
\mathfrak{f}(x)=\mathfrak{s}_{k}(x)+\mathfrak{b}_{k}(x),
$$

where

$\mathfrak{s}_{k}(x)=\frac{1}{2 \pi} \bar{i} \int_{\Gamma_{k}}\left[-\int_{a}^{b}\left(\mathfrak{H}(x, s, \lambda) \Re(s) \mathfrak{f}(s) d s+\mathfrak{K}(\lambda) \int_{a}^{b} d \mathfrak{F}(s, \lambda) \sum_{q=0}^{\mathfrak{\tau}} \lambda^{-q-1} \mathfrak{f}^{(q)}(s) d s\right] d \lambda\right.$,

and

$$
\mathfrak{b}_{k}(x)=\frac{1}{2 \pi i} \int_{\Gamma_{k}} \lambda^{-\tau-1} \int_{a}^{b} \mathfrak{G}(x, s, \lambda) \Re(s) \mathfrak{f}^{(\tau+1)}(s) d s d \lambda .
$$

In view of (10.4), (11.2), (10.1), and (10.3),

where

$$
\mathfrak{s}_{k}(x)=\mathfrak{a}_{0}(x)+\sum_{\beta=1}^{k} \sum_{l=1}^{r_{\beta}} a_{l \beta} \mathfrak{y}^{(l \beta)}(x)
$$

$$
a_{l \beta}=\int_{a}^{b} \mathfrak{Z}^{(l \beta)}(s) \Re(s) \mathfrak{f}(s) d s+\mathfrak{D}_{n-r_{\beta}+l}^{t} \int_{a}^{b} d \mathfrak{F}\left(s, \lambda_{\beta}\right) \sum_{q=0}^{\tau} \lambda_{\beta}^{-q-1} \mathfrak{f}^{(q)}(s) d s
$$

and

The series

$$
\mathfrak{a}_{0}(x)=\operatorname{Res}_{\lambda=0} \mathfrak{K}(\lambda) \int_{a}^{b} d \mathfrak{F}(s, \lambda) \sum_{q=0}^{\tau} \lambda^{-q-1} \mathfrak{f}^{(q)}(s) d s .
$$

$$
\mathfrak{a}_{0}(x)+\sum_{\beta=1}^{\infty} \sum_{l=1}^{r_{\beta}} a_{l \beta} \mathfrak{y}^{(l \beta)}(x)
$$

will be called a formal expansion of $f(x)$. Clearly, $\mathfrak{s}_{k}(x)$ is a partial sum of this series, and the series will converge to $f(x)$ for any value of $x$ for which $\mathfrak{b}_{k}(x)$ converges to zero.

12. Biorthogonality relation. A formal expansion of a given vector was obtained in the previous section without benefit of an orthogonality relation. For the sake of completeness, however, the method of deriving such a relation will be indicated here.

In formula (4.9) let $\lambda$ be the characteristic value $\lambda_{\beta}$, and let $3(x, \lambda)$ be the characteristic vector solution $\mathfrak{z}^{(l \beta)}(x)$ with associated parametric vector $\mathfrak{f}_{l}\left(\lambda_{\beta}\right)$. Also, let $\mathfrak{A}\left(x, \lambda_{\beta}\right)=\lambda_{\beta} \mathfrak{R}(x)+\mathfrak{Q}(x)$. If $\mathfrak{B}(x)$ is replaced by the fundamental matrix solution $\mathfrak{Y}(x, \lambda)$, the formula can be written as

$$
\left(\lambda-\lambda_{\beta}\right) \int_{a}^{b} z^{(\ell \beta)}(x) R(x) Y(x, \lambda)=-\mathfrak{f}_{l}\left(\lambda_{\beta}\right) \int_{a}^{b} d F\left(x, \lambda_{\beta}\right) Y(x, \lambda) .
$$

This is similar to a formula given by Langer [2, (13.7)]. By following Langer's 
analysis, it reduces to a biorthogonality relation which differs from his relation $[2,(13.15)]$ only by having a Stieltjes integral instead of a summation over the discrete boundary points.

13. Convergence of the expansion. Convergence will be established by showing that the vector $\mathfrak{b}_{k}(x)$, defined by (11.4), converges to zero. The following two lemmas will be useful in achieving this end. These lemmas involve a set of variables $t_{1}, t_{2}, \cdots, t_{n}$, which will be represented by the vector symbol $t$. Let the domain of $t_{j}$ be $I_{j}=\left[\alpha_{j}, \beta_{j}\right], j=1,2, \cdots, n$, so that the domain of $t$ is the rectangular region $S$ defined by

$$
S=I_{1} \times I_{2} \times \cdots \times I_{n} .
$$

LEMMA. 13.1 If $\psi(\mathrm{t})$ is a bounded and integrable function on $S$ and if $v_{j}\left(t_{j}\right)$ is a function with total variation $A_{j}$ on $I_{j}, j=1,2, \cdots, n$, then

$$
\left|\int_{I_{n}} \cdots \int_{I_{1}} \psi(\mathrm{t}) d v_{1}\left(t_{1}\right) \cdots d v_{n}\left(t_{n}\right)\right| \leqq L A^{n},
$$

where $L$ is the least upper bound of $|\psi(\mathrm{t})|$ on $S$ and $A$ is the largest of the numbers $A_{1}, A_{2}, \cdots, A_{n}$.

Proof (for $n=2)$. Let $\psi_{2}\left(t_{2}\right)$ be defined by

$$
\psi_{2}\left(t_{2}\right)=\int_{I_{1}} \psi(\mathrm{t}) d v_{1}\left(t_{1}\right)
$$

It is clear that

Therefore

$$
\left|\psi_{2}\left(t_{2}\right)\right| \leqq L A_{1}
$$

$$
\left|\int_{I_{2}} \psi_{2}\left(t_{2}\right) d v_{2}\left(t_{2}\right)\right| \leqq\left(L A_{1}\right) A_{2} \leqq L A^{2},
$$

where $A$ is the larger of $A_{1}$ and $A_{2}$. This proves the lemma when $n=2$ and indicates clearly that the result is valid for any positive integral value of $n$.

LEMMA 13.2. If the components of the matrix $\mathfrak{B}^{*}=\left(v_{i j}^{*}\left(t_{j}\right)\right)$ are of bounded variation and $A$ is an upper bound for the total variation of $v_{i j}^{*}\left(t_{j}\right)$ on $I_{j}$, $i, j=1,2, \cdots, n$, and if $\psi(\mathrm{t})$ is bounded and integrable on $S$, then

$$
\left|\int_{S} \psi(\mathrm{t})\right| d \mathfrak{B}^{*}|| \leqq n ! L A^{n},
$$

where Lis the least upper bound of $\psi(t)$ on $S$.

Proof. The left side of the above relation is an abbreviation for (cf. (7.5) and (7.6)) 


$$
\left|\sum_{\left\{k_{\beta}\right\}} \int_{I_{n}} \cdots \int_{I_{1}} \psi(\mathrm{t}) d v_{k_{1} 1}^{*}\left(t_{1}\right) \cdots d v_{k_{n} n}^{*}\left(t_{n}\right)\right| .
$$

There are $n$ ! terms in the sum, and Lemma 13.1 may be applied to each term. The required result is thereby obtained.

Let $\mathfrak{B}$ be one of the matrices $\mathfrak{B}_{j}$ or $\mathfrak{B}_{i j}, i, j=1,2, \cdots, n$, defined in $\S 7$, and $S$ be the corresponding region $S_{j}$ or $S_{i j}$ associated with it. There exists a nonnegative integer $\theta^{*}$ and a matrix $\mathfrak{B}^{*}$ such that $|\mathfrak{V}|=\lambda^{\theta^{*}}\left|\mathfrak{B}^{*}\right|$, where each component of $\mathfrak{B}^{*}$ is an asymptotic polynomial in $1 / \lambda$, obtained by multiplying the corresponding component of $\mathfrak{B}$ by a nonpositive power of $\lambda$. This representation of $|\mathfrak{B}|$ is not unique, but for each $\mathfrak{B}_{j}$ and $\mathfrak{B}_{i j}$ there will exist a minimum value of $\theta^{*}$ for which the representation is possible. Let the largest of these minimum values (for $i, j=1,2, \cdots, n$ ) be represented by $\theta$, and let it be assumed that for each $\mathfrak{B}$, a matrix $\mathfrak{B}^{*}$ associated with $\theta$ has been determined. Thus, the relation

$$
|\mathfrak{B}|=\lambda^{\theta}|\mathfrak{B} *|
$$

is valid for each $\mathfrak{B}_{j}$ and each $\mathfrak{B}_{i j}$. The components of $\mathfrak{B}^{*}$ are of bounded variation when $|\lambda| \geqq N$. If, therefore, $A$ is an upper bound for the total variation of each component of $\mathfrak{B}^{*}$ and if $\psi$ is restricted as in Lemma 13.2, we have

$$
\left|\int_{S} \psi(\mathrm{t}) e^{-\theta}\right| d \mathfrak{B}|| \leqq n ! L A^{n}, \quad|\lambda| \geqq N
$$

THEOREM 9 (CONVERGENCE THEOREM). If $\mathfrak{f}(x)$ is any vector with a bounded and integrable derivative of order $\tau+1$ and if $\tau>\theta-\rho$, the formal expansion of (11.5) converges uniformly to $f(x)$ on any subinterval of $[a, b]$ on which the boundary problem is regular.

Proof. Substituting in formula (11.4) from (6.12), (7.8), and (7.10), we obtain for the $r$ th component of the vector $\mathfrak{b}_{k}(x)$ (the integers $\rho$ and $\theta$ are those appearing in (8.12) and (13.1), respectively):

$$
b_{k r}(x)=\frac{1}{2 \pi i} \int_{\Gamma_{k}} \int_{a}^{b} \lambda^{\theta-\rho-\tau-1} \phi(x, s, \lambda) d s d \lambda,
$$

where

with

$$
\phi(x, s, \lambda)=\sum_{j=1}^{n} h_{r j}^{(1)}(x, s, \lambda) \eta_{j}(x, s, \lambda)+\sum_{i, j=1}^{n} h_{r i j}^{(2)}(x, s, \lambda) \eta_{i j}(x, s, \lambda),
$$

$$
\begin{aligned}
& h_{r j}^{(1)}(x, s, \lambda)= \pm \sum_{c=1}^{n} p_{r j}(x, \lambda) p^{j c}(s, \lambda) r_{c}(s) f_{c}^{(\tau+1)}(s) \\
& h_{r i j}^{(2)}(x, s, \lambda)= \pm \sum_{c=1}^{n} p_{r i}(x, \lambda) p^{j c}(s, \lambda) r_{c}(s) f_{c}^{(\tau+1)}(s)
\end{aligned}
$$




$$
\begin{aligned}
\eta_{j}(x, s, \lambda) & =\int_{s_{j}} \frac{\exp \left\{\lambda \phi_{j}(x, s)\right\}}{\lambda-\rho D(\lambda)} \lambda^{-\theta}\left|d \mathfrak{B}_{j}\right|, \\
\eta_{i j}(x, s, \lambda) & =\int_{s_{i j}} \frac{\exp \left\{\lambda \phi_{i j}(x, s)\right\}}{\lambda-\rho D(\lambda)} \lambda^{-\theta}\left|d \mathfrak{B}_{i j}\right| .
\end{aligned}
$$

In formulas (13.4) and (13.5), the positive or negative sign is used according as $s<x$ or $s>x$. In (13.6) and (13.7),

$$
S_{j}=\left\{\begin{array}{l}
S_{j}([a, s]), \\
S_{j}([s, b]),
\end{array} \quad S_{i j}=\left\{\begin{array}{l}
S_{i j}([a, s],[s, b]), \\
S_{i j}([s, b],[a, s]),
\end{array}\right.\right.
$$

where the top line, in each case, applies when $s<x$ and the bottom line when $s>x$.

The two functions $\eta_{j}(x, s, \lambda)$ and $\eta_{i j}(x, s, \lambda)$ are similar and may be examined simultaneously by letting the relation

$$
\eta(x, s, \lambda)=\int_{s} \frac{\exp \{\lambda \phi(x, s)\}}{\lambda-\rho D(\lambda)} \lambda^{-\theta}|d \mathfrak{B}|,
$$

represent either (13.6) or (13.7). We may rewrite (13.8) in the form

$$
\eta(x, s, \lambda)=\int_{s} \frac{\exp \left\{\lambda\left(\phi(x, s)-\Omega_{\alpha}\right)\right\}}{\lambda-\rho D(\lambda) \exp \left\{-\lambda \Omega_{\alpha}\right\}} \lambda^{-\theta}|d \mathfrak{B}|,
$$

where $\Omega_{\alpha}$ is a vertex of $P_{D}$. The assumption that $x$ is a point of regularity insures that the functional values of $\phi(x, s)$ over the domain $S$ lie in $P_{D}$. Hence, for every value of $\lambda$, an appropriate choice of $\Omega_{\alpha}$ will insure that

$$
\operatorname{Re}\left\{\lambda\left(\phi(x, s)-\Omega_{\alpha}\right)\right\} \leqq 0 .
$$

It was noted in $\S 8$ that $\lambda^{-\rho} D(\lambda) \exp \left\{-\lambda \Omega_{\alpha}\right\}$ is uniformly bounded from zero for $\lambda$ on any contour of $\left\{\Gamma_{k}\right\}$. Using (13.2), therefore, we infer that $\eta(x, s, \lambda)$ is uniformly bounded in $s$ and $\lambda$ on $\left\{\Gamma_{k}\right\}$.

The $h$-functions, defined by (13.4) and (13.5), are obviously bounded. It follows that $\phi(x, s, \lambda)$ is uniformly bounded. This argument has been made for a fixed point $x$, but if $x$ is any point on an interval of regularity, it can be verified that $\phi(x, s, \lambda)$ is uniformly bounded for $x$ on that interval. Thus, if $\tau>\theta-\rho$, the integrand in formula (13.3) may be represented by $O\left(\lambda^{-2}\right)$. It follows that $\mathfrak{b}_{k r}(x)$ converges uniformly to zero and that the formal expansion converges uniformly to $f(x)$. This proves the theorem.

COROLlary 1. If the formal expansion is differentiated, term by term, to the order $p$ and if $\tau>\theta-\rho+p$, the resulting series converges to the pth derivative of $f(x)$. 
Corollary 2. If $\tau>\theta-\rho+p$ and if a suitable definition is given for $\mathfrak{a}_{0}^{(p)}(x)$, the series

$$
\mathfrak{a}_{0}^{(p)}(x)+\sum_{\beta=1}^{\infty} \sum_{l=1}^{r \beta} a_{l \beta} \lambda_{\beta}^{p} \mathfrak{y}^{(l \beta)}(x)
$$

converges uniformly to $\mathfrak{f}^{(p)}(x)$.

Corollary 3. If $\tau=\theta-\rho$ and $P_{D}$ coincides with $P$, the formal expansion converges uniformly to $f(x)$ on any closed interval in the interior of $[a, b]$.

Proof. By proceeding as in $[1, \S 6$, p. 476$]$, we can show (by using the corollary to Theorem 8) that

$$
\lim _{|\lambda| \rightarrow \infty} \phi(x, s, \lambda)=0,
$$

if $\arg \lambda$ is bounded away from a finite set of rays in the $\lambda$-plane. From this it follows that $\mathfrak{b}_{k r}(x)$ converges uniformly to zero.

14. Remarks. It is a familiar fact that expansions associated with the classical 2-point boundary problem fail, in general, to converge to the given function at the end points of the fundamental interval. In the present case, when the problem is regular on $[a, b]$ and when $\tau>\theta-\rho$, the uniform convergence of the expansion to $f(x)$ on $[a, b]$ is insured by Theorem 9 . The boundary points are not excluded. The following observation is pertinent to this apparent anomally.

Let $\mathfrak{F}(x, \lambda)$ in $(2.1 b)$ be free from $\lambda$ and written as $\mathfrak{F}(x)$. If the boundary integral is applied to the formal expansion, a series is obtained which must converge to $\int_{a}^{b} d \mathfrak{F}(x) \mathfrak{f}(x)$. However, since each characteristic function satisfies the boundary condition, the series reduces to the single term $\int_{a}^{b} d \mathfrak{F}(x) \mathfrak{a}_{0}(x)$. The equality of these two integrals implies that

$$
\int_{a}^{b} d \mathfrak{F}(x)\left(\mathfrak{f}(x)-\mathfrak{a}_{0}(x)\right)=\mathfrak{D} .
$$

The first term in the expansion, therefore, has the effect of modifying $f(x)$ so that it satisfies the boundary condition.

The expansion of a given vector in a series of characteristic solutions of the adjoint system has not been considered here. It may be noted, however, that the discontinuities of these solutions at the boundary points add interest to that expansion problem.

\section{REFERENCES}

1. R. H. Cole, The expansion problem with boundary conditions at a finite set of points, Canad. J. Math. 13 (1961), 462-479.

2. R. E. Langer, The boundary problem of an ordinary linear differential system in the complex domain, Trans. Amer. Math. Soc. 46 (1939), 151-190. 
3. W. C. Sangren, Differential equations with discontinuous coefficients, Oak Ridge National Laboratory Publication, No. 1566 (Physics) (1953).

4. F. W. Stallard, Differential systems with interface conditions, Oak Ridge National Laboratory Report, ORNL 1876 (1955).

5. J. Tamarkin, Some general problems of the theory of ordinary linear differential equations and expansion of an arbitrary function in series of fundamental functions, Math. Z. 27 (1928), 1-54.

6. W. M. Whyburn, Differential equations with general boundary conditions, Bull. Amer. Math. Soc. 48 (1942), 692-704.

7. - Differential systems with general boundary conditions, Seminar Reports in Mathematies, University of California Publications in Mathematics 2 (1944), 45-61.

8. —- Differential systems with boundary conditions at more than two points, Proceedings of the Conference on Differential Equations, University of Maryland (March 17-19, 1955), pp. 1-21, University of Maryland Bookstore, College Park, Md., 1956.

9. C. E. Wilder, Expansion problems of ordinary linear differential equations with auxiliary conditions at more than two points, Trans. Amer. Math. Soc. 18 (1917), 415-442.

The University OF Western ONTARIO, London, Canada 